\title{
4-aminopyridine promotes accelerated skin wound healing
}

2 Mashanipalya G Jagadeeshaprasad ${ }^{1}$, Prem Kumar Govindappa ${ }^{1}$, Amanda M. Nelson ${ }^{2}$, Mark D.

3 Noble $^{3}$ and John C. Elfar ${ }^{1} *$

$4{ }^{1}$ Department of Orthopaedics and Rehabilitation, Center for Orthopaedic Research and

5 Translational Science (CORTS), The Pennsylvania State University College of Medicine,

6 Hershey, PA 17033, USA

$7 \quad{ }^{2}$ Department of Dermatology, The Pennsylvania State University College of Medicine, Hershey, 8 PA 17033, USA.

$9{ }^{3}$ Department of Biomedical Genetics, University of Rochester Stem Cell and Regenerative 10 Medicine Institute, University of Rochester School of Medicine and Dentistry, Rochester, NY 11 14642, USA.

$17 *$ Please address correspondence to:

\section{John C. Elfar, MD, FACS}

19 Department of Orthopaedics and Rehabilitation

20 Center for Orthopaedic Research and Translational Science

21 The Pennsylvania State University College of Medicine and Milton S. Hershey Medical Center

22 Hershey, PA 17033

23 Phone: 717-531-4686; Fax: 717-531-0349

24 E-mail: openelfar@gmail.com and jelfar@pennstatehealth.psu.edu 


\section{Abstract}

31 The discovery of ways to enhance skin wound healing is of great importance due to the

32 frequency of these lesions. We discovered that 4-aminopyridine (4-AP), a potassium channel

33 blocker, greatly enhances skin wound healing. Benefits include faster wound closure, restoration

34 of normal-appearing skin architecture and epidermal thickness, increased vascularization and 35 increases in $\mathrm{K}_{1}{ }^{+}$keratinocytes. Hair follicle number was increased, both histologically and by 36 analysis of K15 and K17 expression. Levels of vimentin (which marks fibroblasts) and $\alpha$ 37 smooth muscle actin ( $\alpha$-SMA, which marks collagen-producing myofibroblasts) increased, as did $38 \alpha-\mathrm{SMA}^{+}$cell numbers. 4-AP also increased numbers of axons and $\mathrm{S}-100^{+}$Schwann cells, and 39 increased expression of p75-NTR and SOX10. Treatment also increased levels of nerve growth 40 factor, transforming growth factor- $\beta$, Substance P and PGP9.5, important modulators of wound 41 healing. As 4-AP is already used for treatment of multiple sclerosis and other chronic 42 neurological syndromes, it has strong potential for rapid translational development.

43

44

45

46

47

48

49

50

51

52

53

54

55

56

57

58

59

60 


\section{Introduction}

Enhancing the healing of skin wounds is an important and challenging medical problem.

Skin wounds are problems in isolation, and also occur concurrently with almost all forms of traumatic injury. Enhancing repair of such lesions is difficult due to the many co-ordinated cellular processes that are required for effective repair. Keratinocyte and fibroblast proliferation and migration are critical in healthy wound healing ${ }^{1-5}$, as are regenerative changes in Schwann cells (SCs) and neurons. For effective repair, a variety of skin cells each need to differentiate along particular pathways, and need to produce extracellular matrix and specific growth factors, such as transforming growth factor- $\beta$ (TGF- $\beta$ ) and nerve growth factor (NGF) ${ }^{1,6-10}$. Skin wound repair also requires re-vascularization and generation of new hair follicles ${ }^{11-13}$. Much is known about the importance of each of these individual components of the repair process, but there is an unmet need for the discovery of clinically relevant agents able to promote regenerative activity of all these processes, which are required for effective skin repair.

While it has been possible to identify multiple cell types and signaling molecules that are important in wound healing, enhancing the healing process is more challenging, particularly so in normal skin. As the major protective barrier between the sterile environment inside the body and the pathogen-rich external world, the evolutionary selective pressure to ensure efficient healing of skin wounds has been very high. Thus, it is not surprising that this is normally an effective process.

Despite the effectiveness of the normal healing response in the skin, several approaches have been identified that improve at least some aspects of this process, thus enabling attention to be focused on several issues relevant to identifying agents most suitable for potential translation. For example, most traumatic injuries are associated with skin wounds, which means that therapeutic agents that can promote healing in more than one tissue are of particular interest. In addition, there is a need for pro-reparative approaches that are suitable for rapid and inexpensive application and for which potential toxicity issues and dosages are well understood. Multiple approaches are of current interest, including pharmacological agents, trophic factors, cell transplantation and genetic manipulation ${ }^{2,6,14-19}$.

Based on studies identifying the importance of components of the peripheral nervous system in skin-wound healing ${ }^{2,8,17}$, and our recent discovery that 4-aminopyridine (4-AP) enhances repair of damage to peripheral nerves ${ }^{20,21}$, we evaluated whether this drug can also 
enhance repair of skin wounds. 4-AP is a potassium channel blocker, and a potential activator of high voltage-activated calcium channels $(\mathrm{HVACCs})^{22}$, with a long history of clinical use for transiently improving neuromuscular function in patients with diverse chronic neurological syndromes ${ }^{23,24}$. In contrast with the effects of 4-AP in chronic syndromes, in which there is no evidence for alterations in tissue structure and where benefits disappear in concurrence with clearance of 4-AP from the body ${ }^{25,26}$, effects in acute injuries include restoration of normal tissue structure and durable functional improvement ${ }^{20,21,27-30}$. However, it is plausible that 4-AP could actually worsen skin wound healing, as several studies have reported that calcium channel blockers enhance repair of such injuries ${ }^{31-33}$, and 4-AP is predicted to increase calcium release.

We now provide evidence that 4-AP treatment of mice with acute skin wounds promotes faster wound healing, extensive changes in wound architecture, and expression of multiple signaling molecules known to be important in healing of such wounds. 4-AP treatment promotes healing-related changes in multiple cell types, including keratinocytes, fibroblasts, myofibroblasts, Schwann cells, neurons and blood vessels. As 4-AP is already approved for human use, our discoveries may enable rapid development of a new approach to enhancing healing of skin wounds with an inexpensive pharmacological approach for which there is extensive existing information on usage in the clinic.

\section{Results}

4-Aminopyridine (4-AP) expedites wound closure and enhances skin regeneration. The beneficial effects of 4-AP on healing of skin wounds were first noticed in studies monitoring wound closure (Fig. 1A-C). We created 5-mm full thickness dorsal excisional wounds in 10-wkold male $\mathrm{C} 57 \mathrm{BL} / 6$ mice $^{34}$; mice were then randomized and treated with either saline or systemic 4- $\mathrm{AP}^{23}$ daily for 14 days. Wounds were splinted with silicone rings to prevent wound contraction and were monitored by digital imaging for morphometry, percentage of wound healing and tissue regeneration on days 3, 5, 7, 9, 12 and 14 post wound (PWD).

We found that the extent of wound closure in 4-AP-treated mice was more than twice that of saline treated mice at PWD3 (day 3: $22.35 \pm 0.30 \%$ vs. $9.88 \pm 0.28 \%$; $<0.0199$ ). Significant differences in wound closure, were seen at every time point examined, including PWD14 (Pvalues $<0.001)$. At this point, 4-AP-treated mice had complete wound closure, while salinetreated mice still showed incomplete healing (day 14: $98.24 \pm 0.10 \%$ vs. $75.28 \pm 0.32 \%$; $\mathrm{P}$ 0.0001) (Fig. 1B and C). 
Analysis of tissue at PWD14 revealed that 4-AP treatment also increased epidermal thickness to that of uninjured tissue (Fig. 1D and E). Wound tissue was analyzed by morphometric analysis on hematoxylin and eosin $(H \& E)$ stained sections ${ }^{1,3,34}$. The newly formed epidermis in 4-AP-treated mice was even slighter thicker than uninjured tissue $(29.74 \pm 1.30 \mu \mathrm{m}$ in 4AP-treated mice vs. $25.41 \pm 0.12 \mu \mathrm{m}$ in uninjured mice; $\mathrm{P}=0.0033$ ), and was significantly thicker than in saline-treated mice $(19.38 \pm 0.96 \mu \mathrm{m} ; \mathrm{P}=0.0067)$.

4-AP treated mice also showed a significant increase in wound-induced hair neogenesis (WIHN) when compared with saline-treated mice (Fig. 1D and F), a feature known to correlate with successful skin regeneration. 4-AP-treated mice showed a 1.8 fold increase in hair follicles over saline-treated mice ( $45 \pm 3$ vs. $25 \pm 3$ follicles/400 $\left.\mu \mathrm{m}^{2} ; \mathrm{P}=0.0067\right)(\text { Fig. } 1 \mathrm{~F})^{35,36}$.

4-AP increases keratinocyte number and epithelial stem-cell markers in healed wounds. We observed 2.3 fold increases in keratin-14 positive $\left(\mathrm{K}_{1} 4^{+}\right)$keratinocytes in the epidermis and in de novo hair follicles of 4-AP treated mice (Fig. 2A) compared with saline-treated mice (cells: $16.65 \pm 0.97 \%$ cells $/ \mathrm{mm}^{2}$ vs. $7.23 \pm 0.73 \%$ cells $/ \mathrm{mm}^{2} ; \mathrm{P}<0.0001$ ) and (protein $-186666667 \pm$ 25271955 integrated density $/ \mathrm{mm}^{2}$ vs. $70298114 \pm 10356742$ integrated density $/ \mathrm{mm}^{2}, \mathrm{P}=0.0063$ ) (Fig. 2B and C). In contrast, expression of K10, a marker of epidermal differentiation, was not impacted by 4-AP treatment at this time point (Supplementary Fig. S1A and B).

In agreement with the increase in hair follicles seen with H\&E staining (Fig. 1D and F), we also saw increases in $\mathrm{K} 7^{+}$and $\mathrm{K} 15^{+}$cells and protein expression, which are markers of hair follicles. We saw a 1.5-fold increase in the percentage of $\mathrm{K}_{1} 7^{+}$cells as a percentage of total DAPI $^{+}$cells (cells: $33.95 \pm 2.80 \%$ cells $/ \mathrm{mm}^{2}$ vs. $22.94 \pm 1.49 \%$ cells $/ \mathrm{mm}^{2} ; \mathrm{P}=0.0014$ ), and in K17 protein (protein: $132329400 \pm 12238815$ integrated density $/ \mathrm{mm}^{2}$ vs. $50989532 \pm 3610166$ integrated density $/ \mathrm{mm}^{2}, \mathrm{P}<0.0001$ ) (Fig. 2D, E and F). Similarly, there were $>115 \%$ increases in $\mathrm{K} 15^{+}$cells (cells: $16.05 \pm 1.38 \%$ cells $/ \mathrm{mm}^{2}$ vs. $7.44 \pm 0.75 \%$ cells $/ \mathrm{mm}^{2}, \mathrm{P}<0.0001$ ), a marker of hair follicle bulge stem cells ${ }^{35,36}$. There were also increases in K15 protein (protein: 71374407 \pm 5506935 integrated density $/ \mathrm{mm}^{2}$ vs. $23022240 \pm 2208956$ integrated density $\left./ \mathrm{mm}^{2} ; \mathrm{P}<0.0001\right)$ (Fig. 2G, H and I).

K14 and K17 expression also increased in the overlying epidermis in both saline and 4AP-treated mice, which is consistent with their known expression following wounding (Fig. 2A and $\mathrm{D})^{37}$. 


\section{4-AP treatment promotes increases in fibroblasts, myofibroblasts and transforming growth} factor- $\boldsymbol{\beta}$ (TGF- $\boldsymbol{\beta})$. Fibroblast migration and maturation contribute to contraction, granulation, and proliferation phases of wound healing. We therefore next examined effects of 4-AP treatment on fibroblasts and on a known regulator of fibroblast differentiation, TGF- $\beta$.

To test whether 4-AP treatment altered fibroblast maturation during wound healing, we first performed Masson's Trichrome staining to examine collagen deposition in the healing wound (Fig 3). This staining revealed elevated collagen deposition in 4-AP treated mice compared to saline treated mice $(\mathrm{P}=0.0025$; Fig. $3 \mathrm{~A}$ and $\mathrm{B})$, with collagen levels like those seen in normal tissue. This staining also revealed a tissue structure and collagen deposition pattern very much like that seen in normal tissue.

4-AP treatment also increased the expression of fibroblast proteins, vimentin and $\alpha$ smooth muscle actin ( $\alpha$-SMA). Immunofluorescence analysis revealed more vimentin ${ }^{+}$ fibroblasts and elevated vimentin levels in wound tissue from 4-AP treated mice than saline treated mice (Fig. 3C and D). We also observed increases in $\alpha$-SMA, which signifies fibroblast differentiation into collagen-producing myofibroblasts ${ }^{1-3}$. Increases were seen in the number of $\alpha$-SMA ${ }^{+}$cells and in $\alpha$-SMA protein $(\mathrm{P}=0.0063$; Fig. $3 \mathrm{C}$ and $\mathrm{E})$.

TGF- $\beta$ plays an important role in promoting myofibroblast differentiation ${ }^{1-3}$, and we found significant increases in TGF- $\beta$ protein expression with 4-AP treatment compared to saline treatment $(\mathrm{P}=0.0001$; Fig. $3 \mathrm{~F}$ and $\mathrm{G})$

\section{4-AP promotes reinnervation and neuropeptide expression. Normal skin wound healing is} also associated with increases in cell division and increases in non-dividing neurons. 4-AP treatment caused increases in both of these measures.

Expression of the proliferation marker, Ki-67, was significantly increased in mice treated with 4-AP compared with saline treated controls. The proportion of $\mathrm{Ki}-67^{+}$cells within hair follicles and epidermis was increased 2.8 fold (cells: $21.87 \pm 2.763$ cells $/ \mathrm{mm}^{2}$ vs. $7.754 \pm$ $1.664 \%$ cells $/ \mathrm{mm}^{2} ; \mathrm{P}=0.0001$ ) (Fig. $4 \mathrm{~A}$ and $\mathrm{B}$ ).

The number of neurons in the skin of 4-AP-treated mice also was increased over that seen in saline-treated animals. Neuronal number was determined by staining with antibodies against high molecular weight neurofilament protein $(\mathrm{NF}-\mathrm{H})^{24,38}$. NF-H axonal counts were increased 2.5 fold (cells: $194 \pm 50.51$ count $/ \mathrm{mm}^{2}$ vs. $74.19 \pm 15.85 \mathrm{count} / \mathrm{mm}^{2} ; \mathrm{P}=0.007$ ) in 4-AP treated mice compared with saline treated controls (Fig. 4A and C). 
We also found that NF-H stained axons in the 4-AP treated mice were more often encountered in direct association with $\mathrm{Ki}-67^{+}$hair follicles (Fig. 4A) than in saline treated controls - an important qualitative finding given that hair follicles are known to be associated with sympathetically innervated arrector pili muscles ${ }^{37}$.

Another example of the ability of 4-AP to restore aspects of skin structure like that seen in uninjured tissue was revealed by staining for protein gene product 9.5 (PGP9.5), a neuronal peptide that promotes wound healing ${ }^{13}$. Fourteen days post wounding, PGP9.5 $5^{+}$nerve fibres in the healed wounds were twice as abundant in 4-AP treated mice, as reflected by increased amounts of PGP 9.5 (protein: $4096938 \pm 713297$ integrated density $/ \mathrm{mm}^{2}$ ), as compared with saline treated mice (protein: $2107970 \pm 325039$ integrated density $\left./ \mathrm{mm}^{2}\right)(\mathrm{P}=0.0012$; Fig. 4D and E). The levels of PGP 9.5 in 4-AP-treated mice were not significantly different from seen in uninjured tissue (Supplementary Fig. S1C and D).

\section{4-AP increases numbers of Schwann cells (SC) and expression of markers of an early} differentiation state. Schwann cells (SC) are critical players in wound healing and are associated with axons around hair-follicles in the wound bed. We also found that the number of SCs was significantly increased in the wounds of 4-AP-treated mice. Analysis of expression of S100, a pan-SC marker ${ }^{1,28}$, identified SCs within both the hypodermis and dermis of the healed wounds (Fig. 5A).

The number of SCs was 3-fold greater in 4-AP treated mice than in saline treated controls (cells: $6.483 \pm 1.163 \%$ cells $/ \mathrm{mm}^{2}$ vs. $2.242 \pm 0.3159 \%$ cells $/ \mathrm{mm}^{2} ; \mathrm{P}=0.0007$ ) (Fig. $5 \mathrm{~A}-\mathrm{B}$ ). SCs were preferentially located around nerve bundles, as predicted by the known affiliation of SCs with nerve cells. 4-AP treatment also increased the expression of p75-NTR (Fig. 5A, C-F), which is thought to be expressed in $\mathrm{SC}-100^{+}$cells as a marker of de-differentiation) $)^{1,39,40}$.

We also found elevated expression of SOX10 and NGF in 4-AP treated mice. SOX10 is required for myelin production in $\mathrm{SCs}^{41}$ and elevated SOX10 expression promotes conversion of mesenchymal cells into p75-NTR expressing neural crest stem cells (NCSC) ${ }^{41,42}$. Conversely, depletion of SOX10 expression significantly delays wound healing and tissue regeneration ${ }^{1}$. NGF plays a significant role in the wound healing process ${ }^{8,38-40,42-44}$ by inducing nerve sprouting from injured nerve endings. NGF also promotes keratinocyte proliferation ${ }^{45}$, and migration of dermal fibroblasts ${ }^{6}$. NGF also acts on non-neuronal cells to sensitize them to substance-P, which 
214 in turns further stimulates more NGF secretion ${ }^{44,46}$ ensuring that keratinocytes, for example, can

215 elaborate and respond to neuronal factors along with neurons.

We found significantly increased SOX10 in both immunofluorescence and western blot analysis (immunofluorescence: $3726901 \pm 151884$ vs. $3125946 \pm 62780$ integrated density/mm²; $\mathrm{P}=0.0018$ and western blot analysis: $6312394 \pm 114415$ vs. $3780312 \pm 437118$ normalized integrated density; $\mathrm{P}=0.0304$ ) (Fig 6A, B, E and F), substance-P (immunofluorescence: $3916297 \pm 247329$ vs. $3120195 \pm 107296$ integrated density $\left./ \mathrm{mm}^{2} ; \mathrm{P}=0.0063\right)(\mathrm{Fig} 6 \mathrm{~A}, \mathrm{C})$, and NGF (4-fold increase- immunofluorescence: $2429872 \pm 375280$ vs. $626967 \pm 101856$ integrated density $/ \mathrm{mm}^{2} ; \mathrm{P}=0.0002$ and western blot analysis: $4337935 \pm 103543$ vs. $2645874 \pm 129171$ normalized integrated density; $\mathrm{P}=0.0005$ ) (Fig 6A, D, E and G) expression in healed wounds

224 from 4-AP treated mice compared to saline treated mice (Fig 6). In all cases, A-AP increased these neuronal molecule to levels which are found in uninjured skin.

\section{4-AP promotes neo-angiogenesis in granulation tissue}

Neo-angiogenesis is necessary to provide nutrients and oxygen to healing wounds. To assess whether 4-AP treatment enhanced wound neo-vascularization, we performed immunofluorescence staining for the endothelial specific marker, CD31 ${ }^{3}$.

We observed larger and more abundant blood vessel networks in dermal tissue from 4AP treated mice than saline treated mice (Supplementary Fig. S1E). Although the number of blood vessels in 4-AP-treated mice was still less than in uninjured tissue, vessels in the newly healed tissue were notably larger. Quantification of CD31 intensity revealed statistically significant increases in blood vessels with 4-AP treatment compared to saline treatment (Supplementary Fig. S1F).

4-AP effectively stimulates proliferation and migration in primary cultures of human skin derived primary cells in-vitro

We next found that effects of 4-AP on keratinocytes, fibroblasts and SCs in-vitro were similar to outcomes observed in-vivo, suggesting 4AP may have direct effects on these cell types. In these experiments, we cultured primary, normal human epidermal keratinocytes $(\mathrm{NHEKs})^{47,48}$, fibroblasts ${ }^{47}$ and dermal $\mathrm{SCs}^{49}$ in the presence or absence of 4-AP.

We first confirmed the purity and identity of each cell type with immunohistochemistry 243 for characteristic markers (Supplementary Fig. S2A-C). There was a modest decrease in MTT 
245 concentrations of 4-AP used in previous work on cells in-vitro ${ }^{24}$ (Supplementary Fig. S2D-F). 246 Automated wound scratch assays were performed ${ }^{19,50,51}$ on confluent monolayers of 247 keratinocytes, fibroblasts, and SCs, with and without $1 \mathrm{mM} 4-\mathrm{AP}^{24,30,52}$, a standard 4-AP dose 248 used in studies in-vitro. 4-AP exposure accelerated scratch closure and keratinocyte migration 249 (Fig. 7A and B; Supplementary Movie 1 and 2) within 3 hours, with complete scratch closure occurring at 18 hours. In contrast, control cultures not exposed to 4-AP closed at 32 hours (Fig. 7A and B).

SOX10 and NGF expression in 4-AP treated keratinocytes were both increased (Fig. 7 CF). Expression of K14 and K17, which are associated with basal, proliferating keratinocytes, were also increased (Supplementary Fig. S3). These data suggest that 4-AP promotes a more proliferative phenotype in keratinocytes, as is necessary for accelerated scratch closure.

In contrast with the effects on keratinocytes, fibroblast migration was unaffected by 4-AP (Supplementary Fig. S4A and B). 4-AP did, however, induce a conversion of fibroblasts to myofibroblasts with increased vimentin and $\alpha$-SMA protein expression (Supplementary Fig. S4C; and Supplementary Movie 3 and Supplementary Movie 4).

In SCs, 4-AP accelerated scratch closure $(80 \%$ by 11 hours with 4-AP treatment compared with 23 hours without treatment) (Supplementary Fig. S5A-B and Supplementary Movie 5 and Supplementary Movie 6). 4-AP treatment also increased SOX10, p75-NTR and NGF expression compared to controls (Supplementary Fig. S5C-E).

We next found that co-culturing cells accelerated scratch culture closure rates and that 4AP exposure further increased these effects. Given that keratinocytes, fibroblasts and SCs cells all interact during wound healing in-vivo, we conducted co-culture experiments with pairs of cell types $^{51}$ with and without 4-AP treatment to determine if 4-AP promotes cellular interactions.

268 Keratinocytes co-cultured with SCs in a ratio mimicking that of epidermal skin (10:1 ratios of keratinocytes:SCs) closed a scratch within 15 hours with 4-AP treatment, which was significantly faster than scratch closure without treatment, which took 20 hours. At 15 hours, the 271 percentage of wound closure was at $98 \%$ in 4-AP treated cultures but only $80 \%$ in non-treated scratch cultures (Fig. 8A and B; and Supplementary Movie 7 and Supplementary Movie 8). 
276

277

278

279

280

281

282

283

284

285

286

287

288

289

290

291

292

293

294

295

296

297

298

299

300

301

302

303

304

305

treated vs. $72 \%$ in non-treated) (Fig. 8C and D; and Supplementary Movie 9 and Supplementary Movie 10).

\section{Discussion}

We found that 4-AP treatment promoted skin wound healing in a standard splinted skin wound model, with multiple beneficial effects including restoration of a normal-appearing skin architecture. In mice with dorsal skin punch lesions, systemic 4-AP treatment caused more rapid wound closure, restoration of normal epidermal thickness, increased vascularization, and increases in $\mathrm{Ki}^{+} 7^{+}$cells and $\mathrm{K}_{14}{ }^{+}$keratinocytes. Hair follicle number was also increased, as determined histologically and by analysis of K15 and K17 expression. Analysis of collagen deposition by Masson's trichrome staining also revealed restoration of normal collagen levels and tissue structure. Levels of vimentin (a marker of fibroblasts) and $\alpha$-SMA (a marker of collagen-producing myofibroblasts) were increased, as were the numbers of $\alpha-\mathrm{SMA}^{+}$cells. Levels of TGF- $\beta$, which promotes myofibroblast differentiation were also increased. Benefits of 4-AP treatment were also seen for multiple aspects of the peripheral nervous system components of skin. The number of neurons was increased in 4-AP treated mice, as determined by counting of NF-H $\mathrm{H}^{+}$axons, as were levels of the neuropeptide PGP 9.5. Treatment with 4-AP also caused increases in numbers of S-100 ${ }^{+} \mathrm{SCs}$, as well as increased expression of p75-NTR, SOX10 and NGF. Finally, 4-AP treatment in-vitro accelerated scratch closure in keratinocytes and SCs, increased SOX10 and NGF expression and also accelerated scratch closure in co-cultures of SCs and keratinocytes, and in co-cultures of keratinocytes and fibroblasts.

Our results extend in a surprising new direction our earlier discoveries that 4-AP exhibits unexpected pro-regenerative properties when used to treat acute traumatic injury in the peripheral nervous system ${ }^{20,27,28}$. We previously found that treatment with 4-AP promotes durable functional recovery and remyelination in crush injuries of sciatic nerves. These findings contrast with the more general use of daily 4-AP treatment to transiently improve neuromuscular function in individuals with chronic multiple sclerosis, nystagmus, spinal cord injury or myasthenia gravis, with benefits that disappear concurrently with 4-AP clearance once treatment is ended ${ }^{53-55}$.

The ability to promote skin wound healing with 4-AP was surprising both because such healing in the skin of mice is already very effective and because of the multiple cell types and 
signaling processes that need to change in a coordinated manner to promote such repair. We observed changes in numbers and behavior of keratinocytes, fibroblasts, Schwann cells, blood vessels, hair follicles and nerve cells, all of which are critical in rebuilding the structure of normal skin ${ }^{1-3,18,36}$. We also observed elevated expression of multiple signaling molecules involved in the regulation of these different cell types. This included increased levels of TGF- $\beta$ (which induces myofibroblast differentiation, keratinocyte proliferation and angiogenesis ${ }^{1,3}$, PGP9.5 (which induces pro-regenerative changes in Schwann cells ${ }^{56}$ and NGF (which promotes neuronal survival and also acts on keratinocytes ${ }^{6,10}$. It is possible that some of the effects of 4-AP are mediated through such molecules as intermediaries, but how 4-AP causes increases in all of these factors is unknown. In-vitro studies also demonstrated direct effects of 4-AP on keratinocytes, SCs and fibroblasts, including increases in SOX10, NGF and $\alpha$-SMA expression and in co-cultures of keratinocytes with SCs or fibroblasts. Although in-vitro studies used concentrations of 4-AP much higher than are achieved in vivo, this is generally the case for in vitro studies ${ }^{24,30,52,57}$. The reason such high 4-AP concentrations are required in vitro is unknown, particularly as results of such studies have accurately predicted the utility of much lower concentrations of 4-AP in clinical situations.

The effects of 4-AP were also surprising because the known effects of 4-AP differ markedly from previous studies on the benefits of inhibiting ion channel function in skin wound healing. In particular, several studies have reported that topical treatment of skin wounds with calcium-channel blockers can promote recovery and potentially decrease scarring in patients ${ }^{31,32}$. These prior findings make the effects of 4-AP somewhat surprising because it is thought that 4AP treatment leads to increased calcium release as an indirect effect of potassium channel inhibition and by direct activation of high voltage-activated calcium channels ${ }^{31,58}$, and 4-AP also is used clinically to treat verapamil overdose ${ }^{59}$. It also has been observed that skin wound healing can be enhanced by inhibition of the kcnh2 potassium channel ${ }^{33}$, but this channel is not known to be a target of 4-AP. As previous studies did not examine the range of cell types and cellular modulators that we studied, it is not possible to determine if these other interventions were as effective as 4-AP. Nonetheless, it appears that ion channel modulation merits further exploration as a means of enhancing healing of skin wounds. 
Regardless of the mechanism(s) by which 4-AP enhances repair of skin wounds, it is noteworthy that the marked enhancement of healing with this compound was also seen in peripheral nerve lesions and in rescue of skeletal muscle atrophy after nerve damage ${ }^{20,29}$. As most traumatic injuries also involve damage to the skin, the ability of a single agent to promote repair in more than one tissue is particularly promising for further development. As 4-AP is already approved for clinical use, and routinely prescribed for individuals with multiple sclerosis, the opportunities to move these discoveries forward to clinical studies seems very high.

\section{MATERIALS AND METHODS}

Study design. The primary objective of this study was to investigate the possible therapeutic effect of 4-AP in enhancing skin wound healing and tissue regeneration in C57BL/6 male mice. All of the animal experimental procedures were performed in accordance with the Penn State College of Medicine institutional guidelines for animal care established by Penn State University and complied with the "Guide for the Care and Use of Laboratory Animals" NIH publication No. 86-23, revised in 2011. The protocol was also approved by Penn State College of Medicine Institutional Animal Care and Use Committee (IACUC NO: PROTO202001314). Mice were age-matched and randomized before treatment into different groups. Data were generated by microscopic analysis of immunohistochemistry, immunofluorescence on fixed skin sections, and immunoblotting of tissue and cell lysates. Sample sizes for each experiment are included in the figure legends.

Wound healing assay. Male C57BL/6 (10 week) mice were purchased from the Jackson Laboratory (Bar Harbor, ME USA). The shaved dorsal skin was folded and raised cranially and caudally at midline to generate two 5-mm wounds using a sterile biopsy punch tool ${ }^{1,3,34}$. A 5mm-diameter silicone ring was then placed and sutured around each wound to restrict contraction. After wound creation and suturing of the silicone ring, wound sites were photographed, and the wound surface was covered with a Tegaderm (3M) sterile transparent dressing. After surgery, mice were administered SR Buprenorphine $(0.05 \mathrm{mg} / \mathrm{kg})$ as postoperative analgesia. The mice were randomized into two groups: a control group (vehicle control), which received $100 \mu \mathrm{l}$ of saline, and a 4-AP group. Mice were treated with 40 $\mu \mathrm{g} / \mathrm{mouse} / \mathrm{daily} 4-\mathrm{AP}(1.6 \mathrm{mg} / \mathrm{kg})$ in $90-110 \mu \mathrm{l}$ of saline intraperitoneally (IP) ${ }^{24,29}$ until day 14 post-wounding. This is a dosage that corresponds with $\sim 40 \%$ of the mouse body surface area 
365

366

367

368

369

370

371

372

373

374

375

376

377

378

379

380

381

382

383

384

385

386

387

388

389

390

391

392

equivalent $^{60}$ of the dosage of $20 \mathrm{mg} /$ day used in treating multiple sclerosis ${ }^{61,62}$ and are less than doses that have been examined in patients with chronic spinal cord injury. Gross wound healing was monitored daily and captured by images taken from day 0 to day 14 (days $0,3,5,7,9,12$ and 14) post-surgery (Fig 1A). Wound areas were measured in pixels using ImageJ-1.53e

Wound healing $(\%)=\frac{(\text { Area of orginal wound at day } 0-\text { Area of wound at postulated day (PWD) })}{\text { Area of orginal wound at day } 0} \times 100$ software (National Institutes of Health, USA) and normalized/corrected for each wound area with reference scales and wound healing expressed as percentage with respect to day 0 wounds ${ }^{1,3,34}$, using the following formula:

Histomorphometry analysis. Wound assessments were conducted at 14 days post-wounding using formalin-fixed paraffin-embedded tissue, serially cut into 5- $\mu \mathrm{m}$ sections on a Microtome (Leica RM2235, Germany). Skin sections were processed for morphometric analysis using hematoxylin and eosin (H\&E) and immunofluorescence staining ${ }^{1,3,34}$. To evaluate the epidermal thickness, and WIHN, four fields of view per sample were imaged by light microscopy (Olympus BX53, Olympus, Tokyo, Japan) at 10 and 40x magnification. Data were averaged for each mouse and then compared between 4-AP and saline treated groups. Collagen formation, maturation and deposition was carried out using Masson's trichrome stain ${ }^{3,36}$ as per manufacturer instructions (Sigma-Aldrich, \# HT15-1KT).

Human primary cell culture experiments. Foreskin collection and preparation human foreskin was rinsed gently with $1 \mathrm{X}-\mathrm{PBS}$ containing antibiotic. The hypodermis and blood vessels were removed. Subsequently, the skin was cut into 1-2 mm pieces and then placed in DMEM medium with dispase-I (Sigma-Aldrich, \# D46693) at $4^{\circ} \mathrm{C}$ for $12-18$ hours. After dispase-I treatment, the epidermis was separated from the dermis ${ }^{48,49}$.

Keratinocyte isolation, culture conditions and characterization - The isolated epidermis was placed in a petri dish containing HBSS buffer (Lonza, \# CC-5022) for 10 minutes at room temperature, then treated with trypsin (Lonza, \# CC-5012) at $37^{\circ} \mathrm{C}$ until the epidermis became loose and the medium cloudy due to keratinocyte release. The cloudy medium was collected and trypsin activity neutralized using fetal bovine serum (FBS, ThermoFisher Scientific \# 10082147)) in 1:1 ratio. The epidermis and suspended keratinocytes were centrifuged at 1500 rpm for 5 minutes. The pellet was resuspended in KGM-GOLD keratinocyte medium (Lonza 
KGM gold and supplements, \# 00192151 and 00192152) ${ }^{47,48}$. The isolated foreskin was cultured at $37^{\circ} \mathrm{C}$ in a $5 \% \mathrm{CO}_{2}$ incubator for 1-2 days to allow keratinocytes to adhere. Adherent keratinocytes were maintained in KGM-Gold medium until cells reached about $80 \%$ confluent.

Dermal Schwann cell isolation, culture conditions and characterization: The separated dermis was minced into small pieces and placed in a petri dish containing collagenase (Gibco, \# 17018029 ) in DMEM basal medium at $37^{\circ} \mathrm{C}$ for 2.5 hours. The dermis was then dissociated cells were collected and centrifuged at $1500 \mathrm{rpm}$ for 5 minutes. The pellet was resuspended in complete DMEM medium, and cells were plated on poly-L-lysine coated dished at $37^{\circ} \mathrm{C}$ in a $5 \% \mathrm{CO}_{2}$ incubator overnight. The next day, adherent cells were treated with $10 \mu \mathrm{M}$ cytosine arabinoside containing DMEM complete medium and incubated at $37^{\circ} \mathrm{C}$ in $5 \% \mathrm{CO}_{2}$ incubator for 24 hours. After this treatment, the cells were cultured in Schwann cell culture medium (ScienCell Research, \#1701 $)^{49}$ until cells reached $\sim 95 \%$ confluency.

Dermal fibroblast isolation, culture conditions and characterization: The separated dermis was minced into small pieces and placed in a petri dish containing collagenase containing DMEM basal medium at $37^{\circ} \mathrm{C}$ for 2.5 hours. The dermis was dissociated the released cells were collected and centrifuged at $1500 \mathrm{rpm}$ for 5 minutes. The pellet was resuspended in complete fibroblast medium (ScienCell Research, \# 2331), and grown on tissue culture dishes $37^{\circ} \mathrm{C}$ in $5 \% \mathrm{CO}_{2}$ incubator overnight ${ }^{47}$. The next day, adherent cells were refed with complete fibroblast medium, and grown at $37^{\circ} \mathrm{C}$ in $5 \% \mathrm{CO}_{2}$ incubator until cells reached $\sim 95 \%$ confluency.

Cell viability assay with 4-aminopyridine. The keratinocytes, Schwann cells and fibroblasts were cultured in 96-well plates for 18 hours. Cells were placed in minimal media (no serum or growth factors) for 4 hours prior to 4-AP treatment. Cells were treated with 4-AP (at concentrations ranging from 1 to $10000 \mu \mathrm{M}$ ) in appropriate cell culture medium for 24

$$
\text { Cell viability }(\%)=\frac{(\mathrm{OD} \text { of } 4-\mathrm{AP} \text { treated cells at particular concentration }-\mathrm{OD} \text { of medium })}{\mathrm{OD} \text { of control cells }(\text { No treatment })-\mathrm{OD} \text { of medium }} \times 100
$$

hours $^{20,24,30,52}$. The cell viability following 4-AP treatment was assayed by MTT assay according to manufacturer's protocols (Roche, cell proliferation kit I (MTT), \# 11465007001). The percentage of live keratinocytes, Schwann cells or fibroblasts was determined using the following formula. 
Cells scratch wound healing migration assay. Keratinocytes, Schwann cells and/or fibroblasts

$421\left(7 \times 10^{4}\right.$ cells/well keratinocytes and $3.5 \times 10^{4}$ cells/well of SCs and FBs $)$, in their respective 422 media, were seeded on tissue culture dishes pre-coated either with collagen-I/PLL on 96-well 423 ImageLock microplates for 6 hours (Incucyte-sartorius plate, \# 4379). For drug treatment, cells were pretreated with 4-AP for 16-18 hours prior to perform the wound scratch assay. Next, wound scratches were created using the IncuCyte automated system (Essen BioScience) $)^{19,50,51}$. After scratching, the cells were washed with PBS and the respective cell media was added with or without $1 \mathrm{mM}$ of $4-\mathrm{AP}^{24,30,52}$. The plate was incubated in the IncuCyte ${ }^{\mathrm{TM}}$ automated imaging system, and wound healing and cell migration was monitored by time-lapse photography capturing images every hour from 0 to 42 hours. The relative area of wound size and cell migration at each time point was analyzed using the IncuCyte ${ }^{\mathrm{TM}}$ Scratch Wound Cell Migration Software Module (Essen BioScience) and the percent of wound healing was calculated from the area measured after scratching relative to the basal area as expressed in pixels.

Immunofluorescence staining of cells. Indirect immunofluorescence analysis was used to identify, characterize and analyze proliferation after 4-AP treatment. For each cell type (keratinocytes, Schwann cells or fibroblasts) treated and untreated cultures were processed in the same experimental session. An equal number of passage 1 cells were seeded on chamber slides. The cells were grown in their respective complete medium in the presence or absence of 4-AP for 72 hours. Cells then were fixed with $4 \%$ paraformaldehyde followed by $0.1 \%$ tritonX-100 and stained with primary antibodies used against, cytokeratin-14, keratin-10, keratin-17, S100, p75-NTR, MPZ, vimentin or $\alpha$-SMA, in 5\% BSA-containing PBS $^{47-49}$. After washing, cells were incubated with respective secondary antibodies and washed with PBS after incubation. After 442 DAPI labeling, the chamber glass slides were mounted using Prolong Gold anti-fade mounting medium and then covered with glass coverslips.

444 Co-culture of keratinocytes with Schwann cells or fibroblast in wound scratch assays. To determine the influence of Schwann cells and fibroblasts on keratinocyte migration and proliferation wound healing scratch assays were conducted ${ }^{51}$. The co-culture experiment was 447 performed with keratinocytes combined with either Schwann cells or fibroblasts in a ratio of 448 10:1. The same propositions of their respective media were added and cells were seeded on 96well ImageLock microplates for 6 hours, in wells pre-coated with collagen-I. For the drug 
450

451

452

453

454

455

456

457

458

459

460

461

462

463

464

465

466

467

468

469

470

471

472

473

474

475

476

477

478

479

treatment, cells were pretreated with 4-AP prior to plating on collagen-coated for 16-18 hours. Next, wound scratches were created and cells were grown with or without 4-AP in the medium. Cells were imaged every hour until 24 hours and analyzed for percent of closure as described above.

Immunohistochemistry and immunofluorescence staining of tissue. Briefly, immunohistochemical and immunofluorescence analysis were performed on 5- $\mu \mathrm{m}$ thick wound healing mouse skin sections. The following primary antibodies were used: mouse Cytokeratin14 antibody (\# ab7800; IF-1:100), rat CD31 antibody (\# 553370; IF-1:100), mouse S100 antibody (\# MA5-12969; IF-1:200), rabbit nerve growth factor receptor - p75-NTR antibody (\# AB1554; IF and WB-1:500), Chicken neurofilament Heavy-NFH antibody (\# NB300-217; IF-1:500), mouse alpha-smooth muscle actin $-\alpha$-SMA antibody (\# 14-9760-82; IF-1:200), mouse TGF3 antibody (\# 27969; IF-1:100), rabbit Ki67 antibody (\# 9129S; IF-1:400), chicken keratin 15 antibody (\# 833901; IF-1:500), rabbit K17 antibody (gift from Pierre Coulombe to AMN; IF1:1000), rabbit interleukin-1 beta antibody (\# GTX74034; IF-1:100), rat F4/80 antibody (\# MCA497GA; IF-1:100), mouse anti-SOX10 antibody (\# sc-365692; IF-1:100 and WB-1:200), chicken anti-myelin basic protein antibody (\# MBP; IF- 1:1000), rabbit vimentin antibody (\# 10366-1-AP; IF-1:200), rabbit NGF antibody (\# MA5-32067; IF-1:100 and WB -1:1000), mouse PGP 9.5 antibody (\# PA5-29012; IF-1:200), chicken anti-P-zero myelin protein antibody (\# PZO; IF-1:200), and rat substance P antibody (\# NB100-65219; IF-1:100). Sections were incubated overnight with 5\% BSA in $0.1 \%$ PBS-T for IF stain and/or 5\% skimmed milk in $0.1 \%$ TBS-T for Western blot at $4^{\circ} \mathrm{C}$, then incubated with secondary antibodies for 1 hour at room temperature. The ProLong ${ }^{\mathrm{TM}}$ Gold Anti-fade Mountant with DAPI (Invitrogen, \# P36935) was used as nuclear counterstain. The immunofluorescence stained sections were imaged using ZEISS Axio Observer 7- Axiocam 506 mono - Apotome. 2 microscope. The image analysis and quantification was carried out using ZEN 2.6 pro (Zeiss) imaging software or ImageJ-1.53e software (National Institutes of Health, USA).

Protein isolation and western blot. For protein isolation the harvested skin tissue was flash frozen immediately. The frozen skin tissue was ground to a fine power using a liquid nitrogen mortar. The harvested cells and /or tissue powder was dissolved in RIPA buffer containing Halt ${ }^{\mathrm{TM}}$ Phosphatase (Thermo Scientific, \# 78420) and Protease Inhibitor Cocktail (Roche 
480

481

482

483

484

485

486

487

488

489

490

491

492

493

494

495

496

497

498

499

500

501

complete tablets mini EASYpack, \# 04694124001). Tissue and cell debris was removed by centrifugation at $14000 \mathrm{rpm}$ for 30 minutes at $4^{\circ} \mathrm{C}$. The supernatants were collected and the total protein concentration was determined by BCA protein assays (Thermo Scientific ${ }^{\mathrm{TM}}$ Pierce $^{\mathrm{TM}}$, \# $23225)$. The proteins $(20-30 \mu \mathrm{g})$ of the tissue protein samples were subjected to $12 \%$ sodium dodecyl sulfate polyacrylamide gel electrophoresis (Bio-Rad mini-PROTEAN TGX Gels, \# 4561044) and transferred to polyvinylidene fluoride (PVDF) membranes. After the membranes were blocked with $5 \%$ non-fat milk in $1 \mathrm{X}$ TBS-T for 1 hour, they were incubated with the appropriate primary antibodies (dilution ranging from 1:200 to $1: 1000$ ) at $4^{\circ} \mathrm{C}$ overnight, then incubated with HRP-conjugated secondary antibodies (dilution,1:3000) for 1 hour. Immunoreactivity was then detected using chemiluminescent substrate (Thermo Scientific ${ }^{\mathrm{TM}}$ SuperSignal ${ }^{\mathrm{TM}}$ West Pico PLUS, \# 34577). The intensities of the bands were quantified using Gel-imaging software (Bio-Rad Laboratories Inc., Image Lab 6.1). The quantified band intensities were normalized using GAPDH and expressed either as normalized intensity or as ratios with respect to saline treated mice.

Statistical analysis. Statistical analysis was performed and graphs created using Prism 9 software (GraphPad Software Inc., San Diego, CA) and all data presented as means \pm SEM. For wound healing multiple time-point comparison studies, Sidak's test, and two-way analysis of variance (ANOVA) was used for analyses. Unpaired t-tests were performed to compare between groups, and one-way analysis of variance (ANOVA) was used for analyses. Sample sizes are indicated in the figure legends. $\mathrm{P}<0.05$ was considered statistically significant for all reported analyses. 


\section{Figures}

\section{Figure 1. 4-Aminopyridine (4-AP) expedites wound closure and enhances skin} regeneration. (A) Schematic illustration of the design of animal experiments to test the beneficial therapeutic effect of 4-AP in C57BL/6 mouse wound splinted model. (B) Representative images of the wound healing in control (saline treated) and 4-AP treated mice at 0, 3, 5, 7, 9, 12 and 14 days after wounding. Scale bar, $1 \mathrm{~mm}$. (C) Percent wound area at each time point relative to the initial wound area in control and 4-AP treated mice. Data showed a significant increase in wound closure from day 3(PWD3) in 4-AP treated compare to control mice. Graph represents mean $\pm \mathrm{SEM}, \mathrm{N}=10$ wounds/group and $* \mathrm{P}=0.01$ to $0.05, * * \mathrm{P}=0.01$ to 0.001 , $* * * \mathrm{P}<0.0002$, and $* * * * \mathrm{P}<0.0001$, two-way ANOVA Sidak's multiple comparisons test.

(D) Representative images of H\&E stained sections of normal control skin and full-thickness excisional wound of saline-control and 4-AP treated skin tissue at day 14 (PWD14). Scale bars = $400 \mu \mathrm{m}$. (E) Quantification of epidermal thickness in H\&E stained sections by ImageJ software; measurements revealed thicker epidermis in 4-AP treated compared to saline-control tissue wounds and 4-AP treated mice epidermis was slightly thicker than that of healthy control uninjured skin. (F) Quantification of the number of de novo hair follicles within healed wounds showed that the mean number of hair follicles was increased in 4-AP treated groups compared to saline-control wound tissues and 4-AP treated mice wound tissue had slightly less number of hair follicles than that of healthy control uninjured skin. Mean $\square \pm \square \mathrm{SEM}, \mathrm{N}=5$ animals skin tissue/group, $* \mathrm{P}=0.01$ to $0.05, * * \mathrm{P}=0.01$ to 0.001 , $* * * \mathrm{P}<0.0002$, and $* * * * \mathrm{P}<0.0001$, unpaired t-test.

\section{Figure 2. 4-AP increases keratinocyte number and epithelial stem-cell markers in healing} wounds. (A) Keratin 14 protein expression in healed epidermis by immunofluorescence analysis. 4-AP treated mice exhibited more intense K14 (green) stain in epidermis and de novo hair follicles compared to control wound mice, nuclear stain DAPI (blue). (B and C) Percent of K14 ${ }^{+}$ cells and K14 protein integrated density in saline-control and 4-AP treated skin wounds at day 14. Scale bars $=50 \mu \mathrm{m}$. Mean $\pm \mathrm{SEM} ; \mathrm{N}=4$ animals tissue wounds/group, $* \mathrm{P}=0.01$ to 0.05 , $* * \mathrm{P}=0.01$ to 0.001 , $* * * \mathrm{P}<0.0002$, and $* * * * \mathrm{P}<0.0001$, unpaired t-test. (D) Representative keratin 17 immunofluorescence images of control and 4-AP treated skin wounds. (E and F) Percent of $\mathrm{K} 17^{+}$cells and $\mathrm{K} 17$ protein integrated density in control and 4-AP treated skin 
532 wounds at day 14; K17 expressing cells were significantly more abundant in the 4-AP treated 533 group than that in the control group. Scale bars $=50 \mu \mathrm{m}$, mean $\pm \mathrm{SEM} ; \mathrm{N}=6$ animals tissue 534 wounds/group, $* \mathrm{P}=0.01$ to $0.05, * * \mathrm{P}=0.01$ to 0.001 , $* * * \mathrm{P}<0.0002$, and $* * * * \mathrm{P}<0.0001$, 535 unpaired t-test. (G) Epithelial hair follicle stem cells measured by immunostaining of K15, 4-AP 536 treated mice showed higher intense K15 (green) stain compared to control wound mice, nuclear 537 stain DAPI (blue). (H and I) Percent of $\mathrm{K} 15^{+}$cells and K15 protein integrated density in control 538 and 4-AP treated skin wounds at day 14. Scale bars $=100 \mu \mathrm{m}$. Mean $\pm \mathrm{SEM} ; \mathrm{N}=4$ animals 539 tissue wounds/group, $* \mathrm{P}=0.01$ to 0.05 , $* * \mathrm{P}=0.01$ to 0.001 , $* * * \mathrm{P}<0.0002$, and $* * * * \mathrm{P}<$ $540 \quad 0.0001$, unpaired t-test.

541 


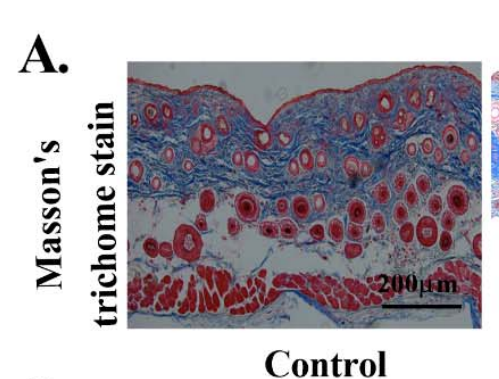

C.

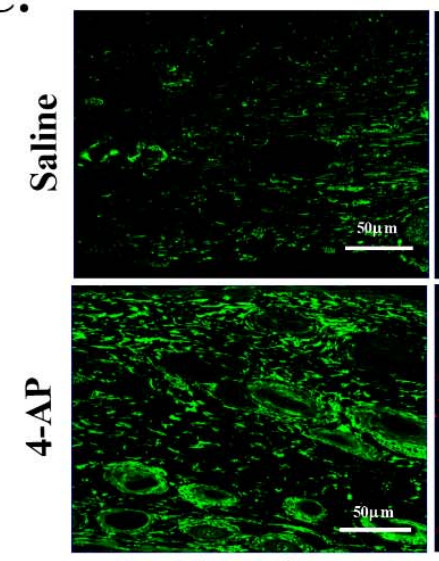

Vimentin
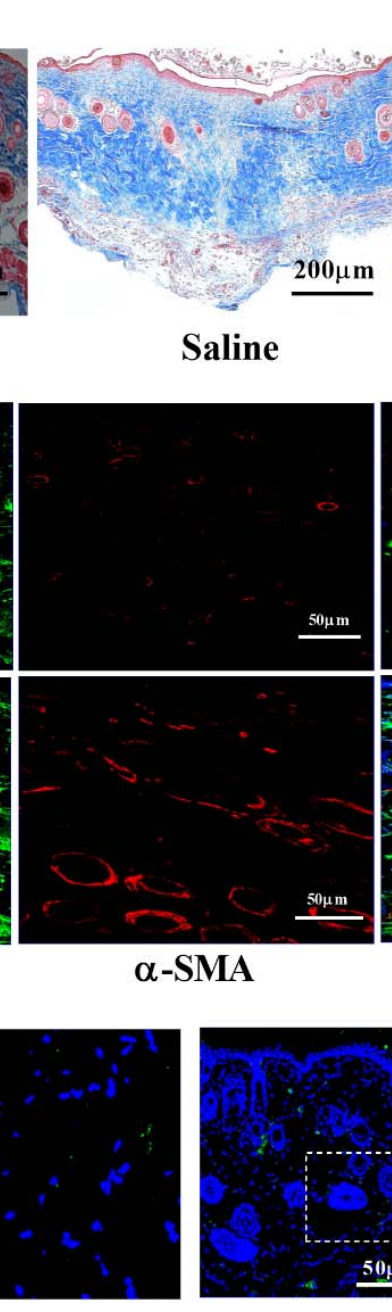

Saline

$\alpha$-SMA

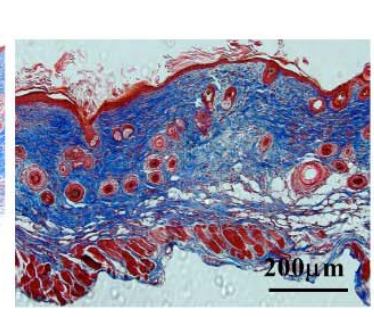

4-AP

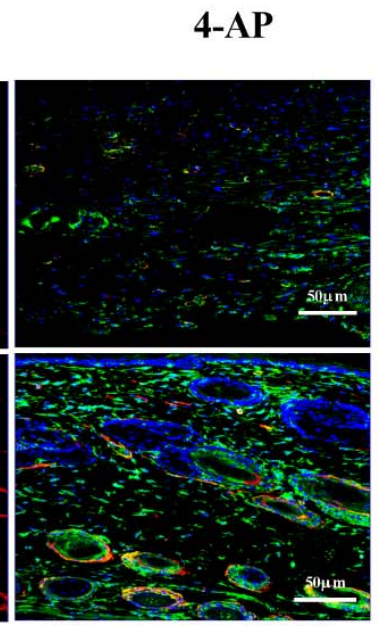

Merge with DAPI
B.

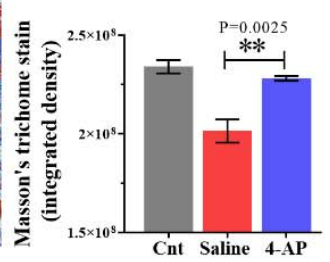

D.

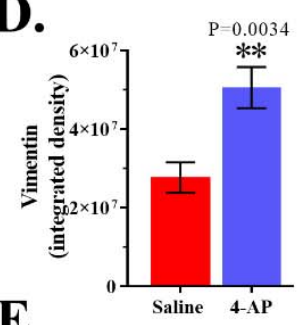

E.

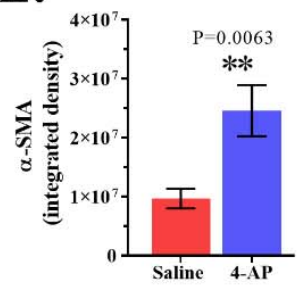

G.

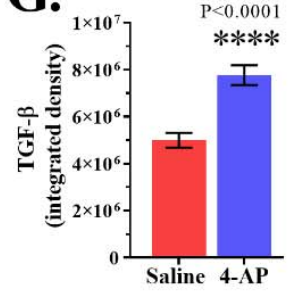

\section{2}

543

544

545

546
Saline
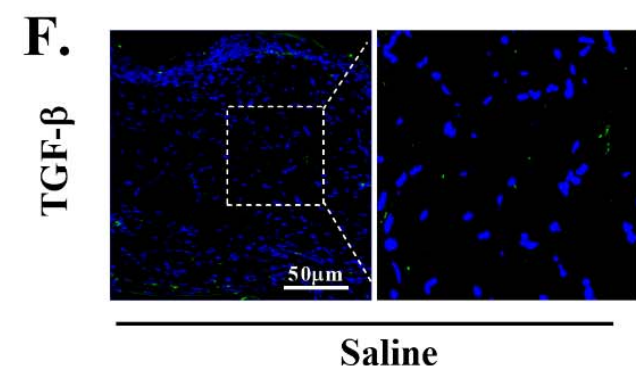

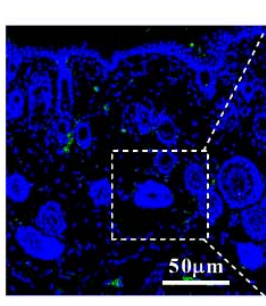

4-AP

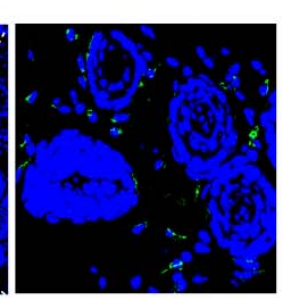

Figure 3. 4-AP treatment increases fibroblast and myofibroblast numbers and levels of transforming growth factor- $\boldsymbol{\beta}$. (A) Representative Masson's trichrome stained images of healing control skin and full-thickness excisional wound of saline-control and 4-AP treated skin tissue at day 14 (PWD14). Scale bars $=200 \square \mu \mathrm{m}$. (B) Collagen density quantified as average blue pixel density per area in wound healing tissue harvested on day 14 (PWD 14). The collagen staining intensity in the 4-AP treated group was significantly higher than that of control. Intensity mean $\square \pm \square \mathrm{SEM}, \mathrm{N}=5 / 6$ animals skin tissue/group, $* \mathrm{P}=0.01$ to $0.05, * * \mathrm{P}=0.01$ to 0.001, $* * * \mathrm{P}<0.0002$, and $* * * * \mathrm{P}<0.0001$, unpaired t-test. (C) Co-immunofluorescence staining of vimentin (green), $\alpha$-SMA (red) and nuclear stain (DAPI-blue) in control and 4-AP treated skin wound sections at day 14. Scale bars $=50 \mu \mathrm{m}$. (D and E) Quantification of vimentin and $\alpha$-SMA protein staining intensity (integrated density), 4-AP treated group showed higher intensity for 
553 both stains compared to saline control group. Mean $\square \pm \square$ SEM, N $=\square 6$ animals wound 554 tissue/group, $* \mathrm{P}=0.01$ to $0.05, * * \mathrm{P}=0.01$ to 0.001 , $* * * \mathrm{P}<0.0002$, and $* * * * \mathrm{P}<0.0001$, unpaired t-test. (F and G) Immunofluorescence of transforming growth factor- $\beta$ (TGF- $\beta$ ) in wound tissue at PWD14. 4-AP treatment increased TGF- $\beta$ expression (green, as detected by immunostaining, nuclei stained with DAPI (blue)). Scale bars $=50 \mu \mathrm{m}$ and TGF- $\beta$ protein quantitative integrated density analysis. Mean $\pm \mathrm{SEM} ; \mathrm{N}=5$ animals wound tissue/group, $* \mathrm{P}=$ 0.01 to $0.05, * * \mathrm{P}=0.01$ to $0.001, * * * \mathrm{P}<0.0002$, and $* * * * \mathrm{P}<0.0001$, unpaired t-test.

560 Figure 4. 4-AP promotes division reinnervation and enhanced PGP9.5 expression. (A) Representative co-immunostaining for $\mathrm{Ki}-67^{+}$cells and $\mathrm{NF}-\mathrm{H}^{+}$cells surrounding de novo hair follicles in the healed wound at day 14. Scale bars $=20 \mu \mathrm{m}$. (B and C) $\mathrm{Ki}^{+}$and NF-H ${ }^{+}$cells were significantly increased in 4-AP-treated wounds. $\mathrm{Ki}^{+} 7^{+}$cells percent and NF-H expression counts was expressed in mean $\pm \mathrm{SEM} ; \mathrm{N}=4$ animal wound tissues/group, $* \mathrm{P}=0.01$ to 0.05 , **P $=0.01$ to $0.001, * * * \mathrm{P}<0.0002$, and $* * * * \mathrm{P}<0.0001$, unpaired t-test. (D) Immunofluorescence staining of healed wound sections for pan-neuronal marker PGP-9.5 (red) and nuclear stain DAPI (blue). Scale bars $=20 \mu \mathrm{m}$. (E) Quantification of PGP-9.5 protein expressing cells with significantly increased PGP-9.5 intensity in 4-AP treated group compared to saline treated group at day 14. Mean $\pm \mathrm{SEM} ; \mathrm{N}=6$ animal wound tissues/group, $* \mathrm{P}=0.01$ to $0.05, * * \mathrm{P}=0.01$ to $0.001, * * * \mathrm{P}<0.0002$, and $* * * * \mathrm{P}<0.0001$, unpaired t-test. p75-NTR (red) in wound sections. Scale bars $=50 \mu \mathrm{m}$. (B and C) Quantification of S100 ${ }^{+}$and p75-NTR ${ }^{+}$expressing cells in healed wounds. 4-AP significantly increased $\mathrm{S}_{100^{+}}$and $\mathrm{p} 75^{+}$cells compared to control. Mean $\pm \mathrm{SEM} ; \mathrm{N}=6$ animal wound tissues/group, $* \mathrm{P}=0.01$ to 0.05 , **P $=$ 0.01 to $0.001, * * * \mathrm{P}<0.0002$, and $* * * * \mathrm{P}<0.0001$, unpaired t-test. (D) Representative western

577 blot of p75-NTR and GAPDH. (E and F) Normalized integrated densities and ratio of p75-NTR 578 protein expression represented as mean $\pm \mathrm{SEM} ; \mathrm{N}=2$ animals wound tissue/group, $* \mathrm{p}<0.05$, 579 unpaired t-test.

580 Figure 6. 4-AP enhanced expression of transcription factors, neurotrophic factors, and 581 neuropeptides associated with reinnervation. (A) Representative images of triple co582 immunostaining of wound skin for the transcription factor SOX10 (green), neuropeptide 583 substance-P (SP - yellow), nerve growth factor (NGF - red), and nuclear stain DAPI (blue). Scale 
584 bars $=50 \mu \mathrm{m}$. (B, C, and D) Quantification of SOX10, substance-P and NGF expressing cells in 585 healed wounds. 4-AP significantly increased intensities of SOX10, substance-P and NGF 586 expressing cells compared to control group on day 14. Data shown as percent mean $\pm \mathrm{SEM} ; \mathrm{N}=$

587 6 animals wound tissue/group, $* \mathrm{P}=0.01$ to 0.05 , ** $\mathrm{P}=0.01$ to 0.001 , *** $\mathrm{P}<0.0002$, and $* * * * \mathrm{P}$ 588 $<0.0001$, unpaired t-test. (E) Representative western blot of SOX10, NGF and GAPDH. (F and G) A representative western blot and normalized integrated densities for SOX10, NGF and GAPDH. Mean \pm SEM; $N=3$ animals wound tissue/group, $* \mathrm{P}=0.01$ to $0.05, * * \mathrm{P}=0.01$ to $0.001, * * * \mathrm{P}<0.0002$, and $* * * * \mathrm{P}<0.0001$, unpaired t-test.

592

Figure 7. 4-AP accelerates in-vitro keratinocyte wound closure. (A) Representative images of in-vitro keratinocyte scratch assays with 4-AP and vehicle control at indicated time points. The yellow lines indicate the wound borders at the beginning of the assay and were recorded every hour until 42 hours. Scale bar $=100 \mu \mathrm{m}$. (B) The relative percentage of wound closure was calculated as the ratio of the remaining wound gap at the given time point compared to time 0 . Mean $\pm \mathrm{SEM}, \mathrm{N}=5$ wound scratch replicates/group, and $* \mathrm{P}=0.01$ to 0.05 , $* * \mathrm{P}=0.01$ to 0.001 , $* * * \mathrm{P}<0.0002$, and $* * * * \mathrm{P}<0.0001$ one-way ANOVA Sidak's multiple comparisons test. (C -F) A representative western blot and normalized integrated densities for SOX10, NGF and GAPDH.

Mean $\pm \mathrm{SEM} ; \mathrm{N}=3$ cell culture replicates/group, ${ }^{*} \mathrm{P}=0.01$ to $0.05, * * \mathrm{P}=0.01$ to 0.001 , $* * * \mathrm{P}<$ 0.0002 , and $* * * * \mathrm{P}<0.0001$, unpaired $\mathrm{t}$-test.

Figure 8. 4-AP treatment enhanced accelerated wound closure in co-culture experiments. assays with 4-AP and vehicle control at indicated time points. The yellow lines indicate the wound borders at the beginning of the assay and were recorded every hour until 24 hours. Scale

611 bar $=100 \mu \mathrm{m}$. (B) The relative percentage of wound closure was calculated as the ratio of the 612 remaining wound gap at the given time point. Mean \pm SEM, $N=5$ wound scratch 
613

614

615

616

617

618

619

620

621

622

623

624

625

626

627

628

629

630

631

632

633

634

635

636

637

638

639

640

641

642

replicates/group, and $* \mathrm{P}=0.01$ to $0.05, * * \mathrm{P}=0.01$ to $0.001, * * * \mathrm{P}<0.0002$, and $* * * * \mathrm{P}<0.0001$ one-way ANOVA Sidak's multiple comparisons test. (C) Representative images in-vitro cocultured keratinocytes and fibroblast scratch assay with 4-AP and vehicle control at indicated time points. The yellow lines indicate the wound borders at the beginning of the assay and were recorded every hour until 24 hours. Scale bar=100 $\mu \mathrm{m}$. (D) The relative percentage of wound closure was calculated as the ratio of the remaining wound gap at the given time point. Mean \pm $\mathrm{SEM}, \mathrm{N}=5$ wound scratch replicates/group, and $* \mathrm{P}=0.01$ to $0.05, * * \mathrm{P}=0.01$ to 0.001 , $* * * \mathrm{P}<$ 0.0002 , and $* * * * \mathrm{P}<0.0001$ one-way ANOVA Sidak's multiple comparisons test.

Acknowledgments: We thank Dr. Hong-Gang Wang and Ms. Xiaoming Liu for allowing us to use the IncuCyte ${ }^{\mathrm{TM}}$ instrument and technical assistance. We would like to thank Dr. Fadia Kamal, Dr. Reyad Elbarbary, and Dr. Srinivas Koduru for allowing us to use lab instruments and technical support. We thank Dr. Pierre A Coulombe for providing the K17 antibody. We would also like to acknowledge staff technical assistance from the Penn State College of Medicine Animal Facility and the Center for Orthopaedic Research and Translational Science (CORTS).

Funding: This work was supported by grants from the NIH (K08 AR060164-01A) and DOD (W81XWH-16-1-0725) to J.C.E. in addition to institutional support from The Pennsylvania State University Hershey Medical Center.

Author contributions: J.C.E. conceived the study. M.G.J., A.M.N., and J.C.E. designed the study. M.G.J., P.K.G., and J.C.E. performed experiments and were involved in the acquisition, data analysis. M.G.J., P.K.G., A.M.N., M.D.N., and J.C.E. involved in the interpretation of data. A.M.N., and J.C.E. contributed reagents/materials/analysis tools. M.G.J., A.M.N., M.D.N., and J.C.E. wrote the manuscript. All the authors read the manuscript and have given approval to the final version of the manuscript.

Conflicts of Interest: M.G.J., P.K.G., J.C.E., are inventors on patent/patent application (63/005,890 and 63/133,407 and PCT/US21/14442) submitted by Pennsylvania State University that covers "Method and materials for treating nerve injury and/or wound healing" and "Therapeutic treatments for hair growth". J.C.E. and M.D.N. are founding members of Peripheral Therapeutics, a company that has licenced a previous patent on the use of 4-AP for traumatic peripheral nerve injury and nerve continuity diagnosis. All other authors declare that they have no competing financial interests. 
643 Data and supplementary materials availability: All data associated with this study are

644 described and shown in the paper or the Supplementary Materials.

645

646

647

648

649

650

651

652

653

654

655

656

657

658

659

660

661

662

663

664

665

666

667

668

669

670

671

672

673

674

675

676

677

678

679

680

681

682

683

684

685

\section{References:}

1 Parfejevs, V. et al. Injury-activated glial cells promote wound healing of the adult skin in mice. Nat Commun 9, 236, doi:10.1038/s41467-017-01488-2 (2018).

2 Lebonvallet, N., Laverdet, B., Misery, L., Desmouliere, A. \& Girard, D. New insights into the roles of myofibroblasts and innervation during skin healing and innovative therapies to improve scar innervation. Exp Dermatol 27, 950-958, doi:10.1111/exd.13681 (2018).

3 Yeh, C. J. et al. The effects of artocarpin on wound healing: in vitro and in vivo studies. Scientific reports 7, 15599, doi:10.1038/s41598-017-15876-7 (2017).

4 Pastar, I. et al. Epithelialization in Wound Healing: A Comprehensive Review. Adv Wound Care (New Rochelle) 3, 445-464, doi:10.1089/wound.2013.0473 (2014).

5 Yang, R. et al. Epidermal stem cells in wound healing and their clinical applications. Stem Cell Res Ther 10, 229, doi:10.1186/s13287-019-1312-z (2019).

6 Chen, J. C. et al. NGF accelerates cutaneous wound healing by promoting the migration of dermal fibroblasts via the PI3K/Akt-Rac1-JNK and ERK pathways. BioMed research international 2014, 547187, doi:10.1155/2014/547187 (2014).

7 Lee, S. et al. NGF-TrkA signaling dictates neural ingrowth and aberrant osteochondral differentiation after soft tissue trauma. Nat Commun 12, 4939, doi:10.1038/s41467-021-25143-z (2021).

8 Cheret, J., Lebonvallet, N., Carre, J. L., Misery, L. \& Le Gall-lanotto, C. Role of neuropeptides, neurotrophins, and neurohormones in skin wound healing. Wound Repair Regen 21, 772-788, doi:10.1111/wrr.12101 (2013).

9 Ashrafi, M., Baguneid, M. \& Bayat, A. The Role of Neuromediators and Innervation in Cutaneous Wound Healing. Acta dermato-venereologica 96, 587-594, doi:10.2340/00015555-2321 (2016).

10 Palazzo, E. et al. Role of neurotrophins on dermal fibroblast survival and differentiation. J Cell Physiol 227, 1017-1025, doi:10.1002/jcp.22811 (2012).

11 Nguyen, V. T. et al. Delayed Healing of Sickle Cell Ulcers Is due to Impaired Angiogenesis and CXCL12 Secretion in Skin Wounds. J Invest Dermatol 136, 497-506, doi:10.1016/j.jid.2015.11.005 (2016).

12 Gurtner, G. C., Werner, S., Barrandon, Y. \& Longaker, M. T. Wound repair and regeneration. Nature 453, 314-321, doi:10.1038/nature07039 (2008).

$13 \mathrm{Wu}$, Y., Chen, L., Scott, P. G. \& Tredget, E. E. Mesenchymal stem cells enhance wound healing through differentiation and angiogenesis. Stem Cells 25, 2648-2659, doi:10.1634/stemcells.2007-0226 (2007).

14 Whittam, A. J. et al. Challenges and Opportunities in Drug Delivery for Wound Healing. Adv Wound Care (New Rochelle) 5, 79-88, doi:10.1089/wound.2014.0600 (2016).

15 Dixit, S. et al. Immunological challenges associated with artificial skin grafts: available solutions and stem cells in future design of synthetic skin. J Biol Eng 11, 49, doi:10.1186/s13036-0170089-9 (2017).

16 Woodrow, T., Chant, T. \& Chant, H. Treatment of diabetic foot wounds with acellular fish skin graft rich in omega-3: a prospective evaluation. $J$ Wound Care 28, 76-80, doi:10.12968/jowc.2019.28.2.76 (2019). 
Silva, W. N. et al. Role of Schwann cells in cutaneous wound healing. Wound Repair Regen 26, 392-397, doi:10.1111/wrr.12647 (2018).

18 Matsuda, H. et al. Role of nerve growth factor in cutaneous wound healing: accelerating effects in normal and healing-impaired diabetic mice. J Exp Med 187, 297-306, doi:10.1084/jem.187.3.297 (1998).

19 Gostynska, N. et al. The pleiotropic molecule NGF regulates the in vitro properties of fibroblasts, keratinocytes, and endothelial cells: implications for wound healing. Am J Physiol Cell Physiol 318, C360-C371, doi:10.1152/ajpcell.00180.2019 (2020).

20 Tseng, K. C. et al. 4-Aminopyridine promotes functional recovery and remyelination in acute peripheral nerve injury. EMBO molecular medicine 8, 1409-1420, doi:10.15252/emmm.201506035 (2016).

21 Noble, M., Tseng, K. C., Li, H. \& Elfar, J. C. 4-Aminopyridine as a Single Agent Diagnostic and Treatment for Severe Nerve Crush Injury. Mil Med 184, 379-385, doi:10.1093/milmed/usy399 (2019).

22 Wu, Z. Z., Li, D. P., Chen, S. R. \& Pan, H. L. Aminopyridines potentiate synaptic and neuromuscular transmission by targeting the voltage-activated calcium channel beta subunit. $J$ Biol Chem 284, 36453-36461, doi:10.1074/jbc.M109.075523 (2009).

23 Jensen, H. B., Ravnborg, M., Dalgas, U. \& Stenager, E. 4-Aminopyridine for symptomatic treatment of multiple sclerosis: a systematic review. Ther Adv Neurol Disord 7, 97-113, doi:10.1177/1756285613512712 (2014).

24 Dietrich, M. et al. Protective effects of 4-aminopyridine in experimental optic neuritis and multiple sclerosis. Brain 143, 1127-1142, doi:10.1093/brain/awaa062 (2020).

25 Khammy, M. M. et al. 4-Aminopyridine: a pan voltage-gated potassium channel inhibitor that enhances Kv 7.4 currents and inhibits noradrenaline-mediated contraction of rat mesenteric small arteries. Br J Pharmacol 175, 501-516, doi:10.1111/bph.14097 (2018).

26 Goodman, A. D. et al. A phase 3 trial of extended release oral dalfampridine in multiple sclerosis. Annals of neurology 68, 494-502, doi:10.1002/ana.22240 (2010).

27 Manoukian, O. S., Arul, M. R., Rudraiah, S., Kalajzic, I. \& Kumbar, S. G. Aligned microchannel polymer-nanotube composites for peripheral nerve regeneration: Small molecule drug delivery. J Control Release 296, 54-67, doi:10.1016/j.jconrel.2019.01.013 (2019).

28 Manoukian, O. S. et al. Biopolymer-nanotube nerve guidance conduit drug delivery for peripheral nerve regeneration: In vivo structural and functional assessment. Bioact Mater 6, 2881-2893, doi:10.1016/j.bioactmat.2021.02.016 (2021).

29 Yue, L. et al. 4-Aminopyridine attenuates muscle atrophy after sciatic nerve crush injury in mice. Muscle \& nerve, doi:10.1002/mus.26516 (2019).

30 Bei, F. et al. Restoration of Visual Function by Enhancing Conduction in Regenerated Axons. Cell 164, 219-232, doi:10.1016/j.cell.2015.11.036 (2016).

31 Ashkani-Esfahani, S. et al. Verapamil, a Calcium-Channel Blocker, Improves the Wound Healing Process in Rats with Excisional Full-Thickness Skin Wounds Based on Stereological Parameters. Adv Skin Wound Care 29, 271-274, doi:10.1097/01.ASW.0000488666.03896.e6 (2016).

32 Boggio, R. F., Boggio, L. F., Galvao, B. L. \& Machado-Santelli, G. M. Topical verapamil as a scar modulator. Aesthetic Plast Surg 38, 968-975, doi:10.1007/s00266-014-0400-9 (2014).

33 Zhang, W. \& Bei, M. Kcnh2 and Kcnj8 interactively regulate skin wound healing and regeneration. Wound Repair Regen 23, 797-806, doi:10.1111/wrr.12347 (2015).

34 Chen, L., Mirza, R., Kwon, Y., DiPietro, L. A. \& Koh, T. J. The murine excisional wound model: Contraction revisited. Wound Repair Regen 23, 874-877, doi:10.1111/wrr.12338 (2015).

35 Ito, M. et al. Wnt-dependent de novo hair follicle regeneration in adult mouse skin after wounding. Nature 447, 316-320, doi:10.1038/nature05766 (2007). 
73436 Nelson, A. M. et al. dsRNA Released by Tissue Damage Activates TLR3 to Drive Skin Regeneration. Cell stem cell 17, 139-151, doi:10.1016/j.stem.2015.07.008 (2015).

37 Shwartz, Y. et al. Cell Types Promoting Goosebumps Form a Niche to Regulate Hair Follicle Stem Cells. Cell 182, 578-593 e519, doi:10.1016/j.cell.2020.06.031 (2020). Wilfong, E. R. \& Dey, R. D. Nerve growth factor and substance P regulation in nasal sensory neurons after toluene diisocyanate exposure. Am J Respir Cell Mol Biol 30, 793-800, doi:10.1165/rcmb.2003-03030C (2004). 801, doi:10.1177/0300985818781930 (2018). Wong, S. T. et al. A p75(NTR) and Nogo receptor complex mediates repulsive signaling by myelin-associated glycoprotein. Nature neuroscience 5, 1302-1308, doi:10.1038/nn975 (2002). Motohashi, T. et al. Sox10 Functions as an Inducer of the Direct Conversion of Keratinoc
Neural Crest Cells. Stem Cells Dev 29, 1510-1519, doi:10.1089/scd.2020.0106 (2020). development. The Journal of neuroscience : the official journal of the Society for Neuroscience 20, 7706-7715 (2000).

43 Raychaudhuri, S. K., Raychaudhuri, S. P., Weltman, H. \& Farber, E. M. Effect of nerve growth factor on endothelial cell biology: proliferation and adherence molecule expression on human dermal microvascular endothelial cells. Arch Dermatol Res 293, 291-295, doi:10.1007/s004030100224 (2001).

44 Wei, T. et al. Keratinocyte expression of inflammatory mediators plays a crucial role in substance P-induced acute and chronic pain. J Neuroinflammation 9, 181, doi:10.1186/1742-2094-9-181 (2012).

45 Paus, R., Luftl, M. \& Czarnetzki, B. M. Nerve growth factor modulates keratinocyte proliferation in murine skin organ culture. Br J Dermatol 130, 174-180, doi:10.1111/j.13652133.1994.tb02896.x (1994). Vitro and In Vivo under Hypoxia. Biomedicines 9, doi:10.3390/biomedicines9020222 (2021).

47 Henrot, P. et al. A Method for Isolating and Culturing Skin Cells: Application to Endothelial Cells, Fibroblasts, Keratinocytes, and Melanocytes From Punch Biopsies in Systemic Sclerosis Skin. Front Immunol 11, 566607, doi:10.3389/fimmu.2020.566607 (2020).

48 McHeik, J. N. et al. Foreskin-isolated keratinocytes provide successful extemporaneous autologous paediatric skin grafts. J Tissue Eng Regen Med 10, 252-260, doi:10.1002/term.1690 (2016).

49 Stratton, J. A. et al. Purification and Characterization of Schwann Cells from Adult Human Skin and Nerve. eNeuro 4, doi:10.1523/ENEURO.0307-16.2017 (2017).

50 Riis, S. et al. Hypoxia enhances the wound-healing potential of adipose-derived stem cells in a novel human primary keratinocyte-based scratch assay. Int J Mol Med 39, 587-594, doi:10.3892/ijmm.2017.2886 (2017).

51 Wang, Z. et al. Coculture with human fetal epidermal keratinocytes promotes proliferation and migration of human fetal and adult dermal fibroblasts. Molecular medicine reports 11, 11051110, doi:10.3892/mmr.2014.2798 (2015).

52 Kirchhoff, C., Leah, J. D., Jung, S. \& Reeh, P. W. Excitation of cutaneous sensory nerve endings in the rat by 4-aminopyridine and tetraethylammonium. J Neurophysiol 67, 125-131, doi:10.1152/jn.1992.67.1.125 (1992).

781 multiple sclerosis. Annals of neurology 27, 186-192, doi:10.1002/ana.410270215 (1990). 
54 Lundh, H., Nilsson, O. \& Rosen, I. Effects of 4-aminopyridine in myasthenia gravis. Journal of neurology, neurosurgery, and psychiatry 42, 171-175, doi:10.1136/jnnp.42.2.171 (1979).

55 Hansebout, R. R., Blight, A. R., Fawcett, S. \& Reddy, K. 4-Aminopyridine in chronic spinal cord injury: a controlled, double-blind, crossover study in eight patients. Journal of neurotrauma 10, 1-18, doi:10.1089/neu.1993.10.1 (1993).

56 Cheret, J. et al. Sensory Reinnervation of Human Skin by Human Neural Stem CellDerived Peripheral Neurons Ex Vivo. J Invest Dermatol, doi:10.1016/j.jid.2021.05.032 (2021).

57 Bostock, H., Sears, T. A. \& Sherratt, R. M. The effects of 4-aminopyridine and tetraethylammonium ions on normal and demyelinated mammalian nerve fibres. J Physio/ 313, 301-315 (1981).

58 Bhaskar, H. N., Udupa, S. L. \& Udupa, A. L. Effect of nifedipine and amlodipine on wound healing in rats. Indian J Physiol Pharmacol 48, 111-114 (2004).

59 Hofer, C. A., Smith, J. K. \& Tenholder, M. F. Verapamil intoxication: a literature review of overdoses and discussion of therapeutic options. Am J Med 95, 431-438, doi:10.1016/00029343(93)90314-f (1993).

60 Reagan-Shaw, S., Nihal, M. \& Ahmad, N. Dose translation from animal to human studies revisited. The FASEB journal : official publication of the Federation of American Societies for Experimental Biology 22, 659-661, doi:10.1096/fj.07-9574LSF (2008).

61 Dunn, J. \& Blight, A. Dalfampridine: a brief review of its mechanism of action and efficacy as a treatment to improve walking in patients with multiple sclerosis. Current medical research and opinion 27, 1415-1423, doi:10.1185/03007995.2011.583229 (2011).

62 Krishnan, A. V. \& Kiernan, M. C. Sustained-release fampridine and the role of ion channel dysfunction in multiple sclerosis. Multiple sclerosis (Houndmills, Basingstoke, England) 19, 385391, doi:10.1177/1352458512463769 (2013). 


\section{4-aminopyridine promotes accelerated skin wound healing}

Mashanipalya G Jagadeeshaprasad ${ }^{1}$, Prem Kumar Govindappa ${ }^{1}$, Amanda M. Nelson ${ }^{2}$, Mark D. Noble $^{3}$ and John C. Elfar ${ }^{1 *}$

${ }^{1}$ Department of Orthopaedics and Rehabilitation, Center for Orthopaedic Research and Translational Science (CORTS), The Pennsylvania State University College of Medicine, Hershey, PA 17033, USA

${ }^{2}$ Department of Dermatology, The Pennsylvania State University College of Medicine, Hershey, PA 17033, USA.

${ }^{3}$ Department of Biomedical Genetics, University of Rochester Stem Cell and Regenerative Medicine Institute, University of Rochester School of Medicine and Dentistry, Rochester, NY 14642, USA.

* Please address correspondence to:

John C. Elfar, MD, FACS

Department of Orthopaedics and Rehabilitation Center for Orthopaedic Research and Translational Science

The Pennsylvania State University College of Medicine and Milton S. Hershey Medical Center Hershey, PA 17033

Phone: 717-531-4686; Fax: 717-531-0349

E-mail: openelfar@gmail.com and jelfar@pennstatehealth.psu.edu 


\section{Supplementary Information}

\section{Supplementary Figures}

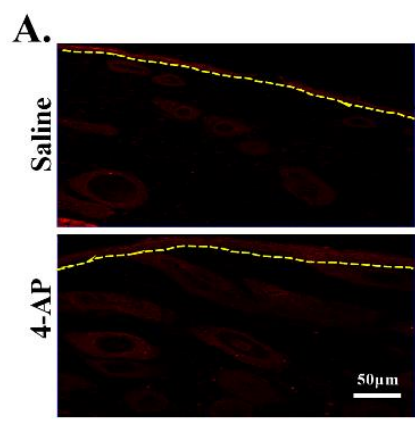

K10

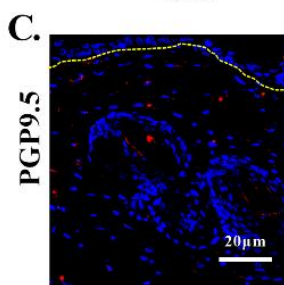

Control

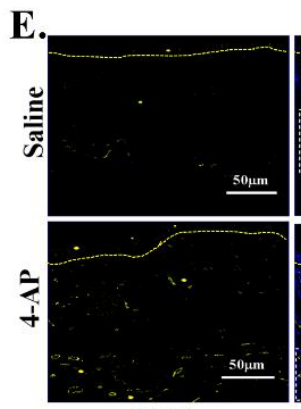

CD31

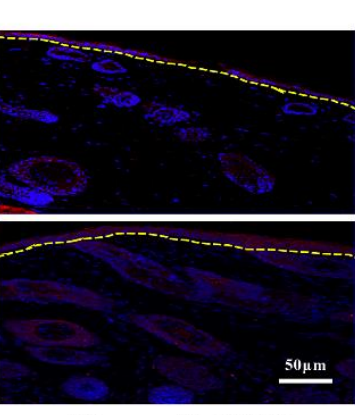

Merge with DAPI

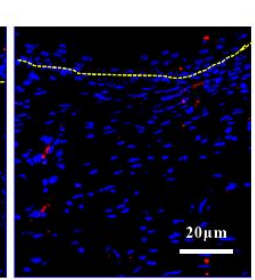

Saline

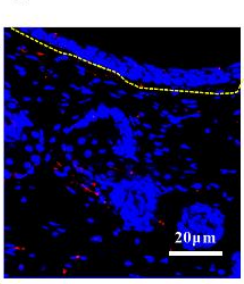

4-AP

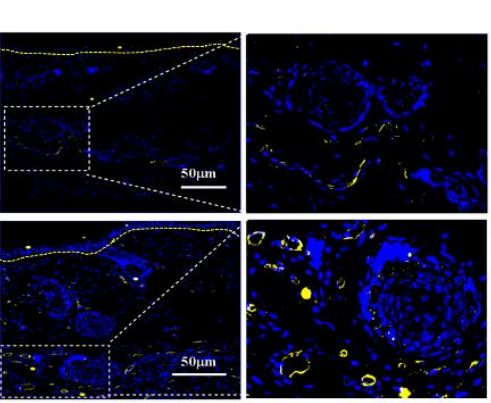

CD31 merge with DAPI
B.

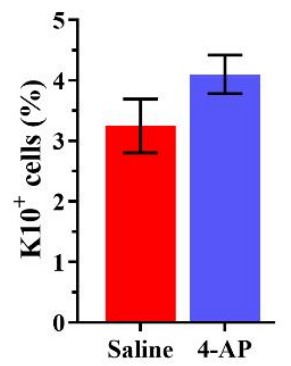

D.

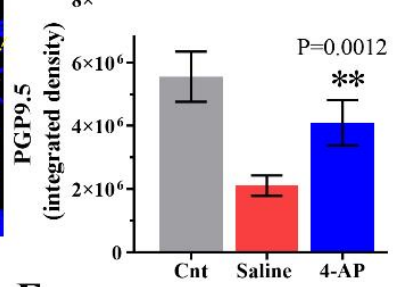

F.

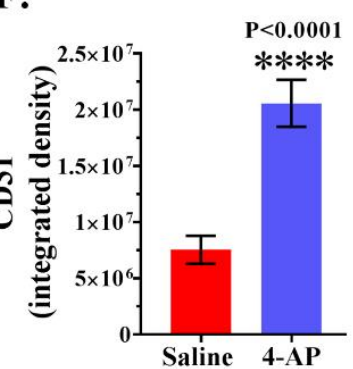

Supplementary Fig. S1. 4-AP induced neo-angiogenesis and neuronal peptide wound healing and did not alter keratinocyte K10 expression. (A) Keratin 10 protein expression in healed epidermis by immunofluorescence. 4-AP treatment did not cause any change in expression of keratinocyte K10 expression. Scale bars $=50 \mu \mathrm{m}$. (B) Percent of K10 $0^{+}$cells in control and 4-AP treated skin wounds at day 14 . Mean $\pm \mathrm{SEM} ; \mathrm{N}=4$ animals wound tissue/group and $* \mathrm{P}=0.01$ to $0.05, * * \mathrm{P}=0.01$ to $0.001, * * * \mathrm{P}<0.0002$, and $* * * * \mathrm{P}<0.0001$ unpaired $\mathrm{t}$-test.

Immunofluorescence staining of control and healed wound sections for pan-neuronal marker PGP9.5 (red) and nuclear stain DAPI (blue). Scale bars $=20 \mu \mathrm{m}$. (D) Quantification of PGP-9.5 protein expressing cells showed significantly increased PGP-9.5 intensity in the 4-AP treated group compared to the saline treated group at day 14. PGP 9.5 in 4-AP-treated mice was not significantly different from seen in uninjured (control) tissue. Mean \pm SEM; $N=6$ animal wound tissues/group 
and 4 control tissue, $* \mathrm{P}=0.01$ to $0.05, * * \mathrm{P}=0.01$ to $0.001, * * * \mathrm{P}<0.0002$, and $* * * * \mathrm{P}<0.0001$, unpaired t-test. $(\mathbf{E}) \mathrm{CD} 31$ protein expression in healed skin by immunofluorescence. Scale bars $=$ $100 \mu \mathrm{m}$. (F) Quantification of CD31 staining intensity, 4-AP treated group showed higher intensity compared to saline control group. Mean $\pm \mathrm{SEM}, \mathrm{N}=6$ animals wound tissue/group and $* \mathrm{P}=0.01$ to $0.05, * * \mathrm{P}=0.01$ to $0.001, * * * \mathrm{P}<0.0002$, and $* * * * \mathrm{P}<0.0001$ unpaired t-test. 


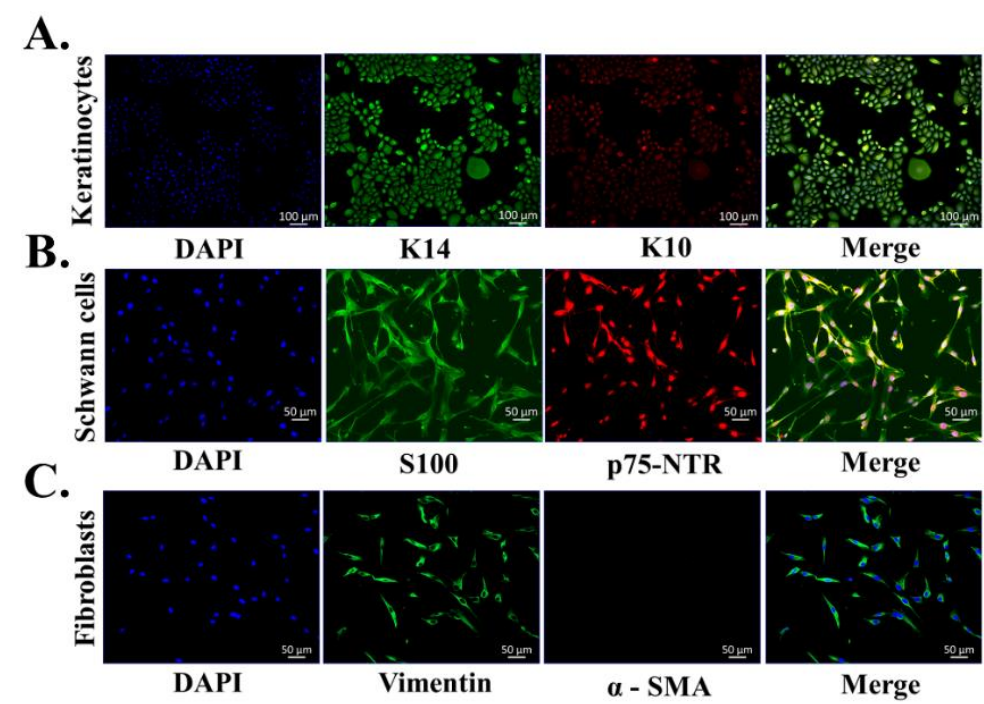

D.

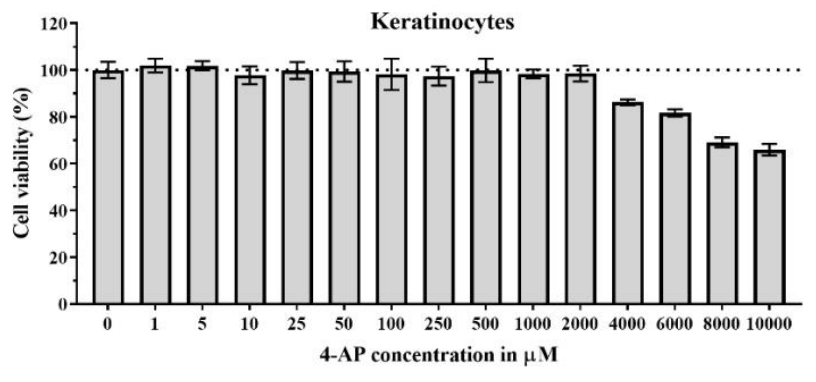

E.

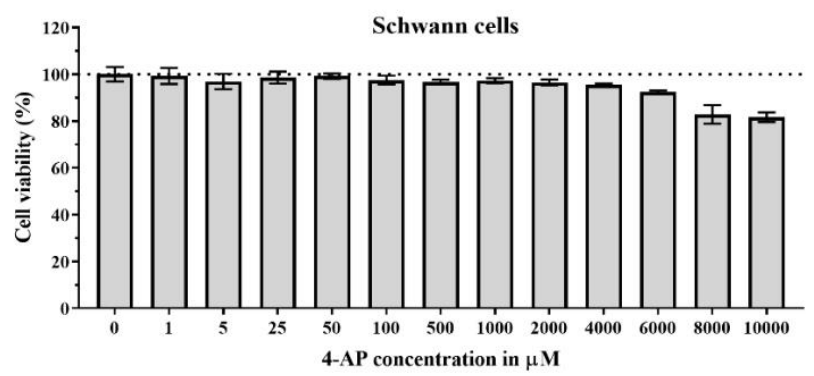

F.

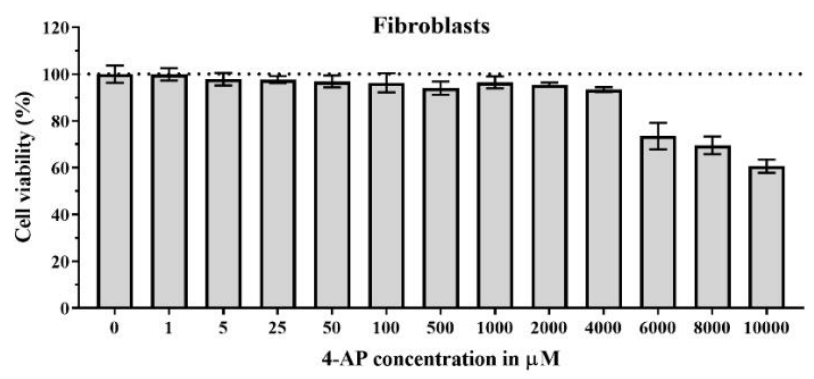

Supplementary Fig. S2. Isolation and characterization of human skin derived primary keratinocytes, Schwann cells, and fibroblasts. (A) Keratinocytes were characterized using keratin 14 (K14, a marker of proliferative keratinocytes) and K10 (keratin 10, a marker of 
keratinocyte differentiation marker). Scale bars $=100 \mu \mathrm{m}$. (B) Schwann cells were identified using S100 (Schwann cells marker) and p75-NTR (nerve growth factor receptor marker). Scale bars = $50 \mu \mathrm{m}$. (C) Fibroblasts were characterized using vimentin (fibroblast marker) and $\alpha$-smooth muscle actin (fibroblast differentiation, myofibroblast marker). Scale bars, $50 \mu \mathrm{m}$. (D-F) Cell viability using MTT assay with different concentrations of 4-AP (ranging from 1 to $10000 \mu \mathrm{M}$ ) for (D) Keratinocytes, (F) Schwann cells and (F) fibroblast. Mean \pm SEM, N=3 replicates/concentration of 4-AP. 


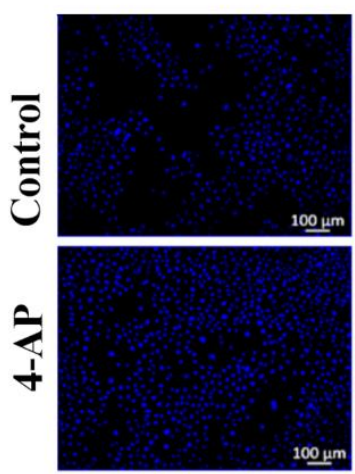

DAPI
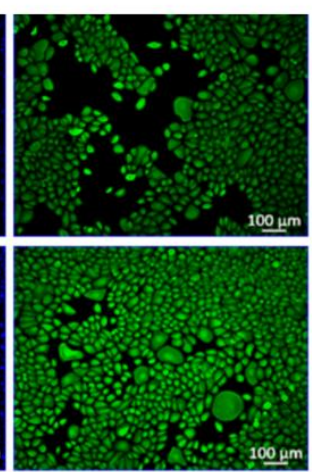

K14
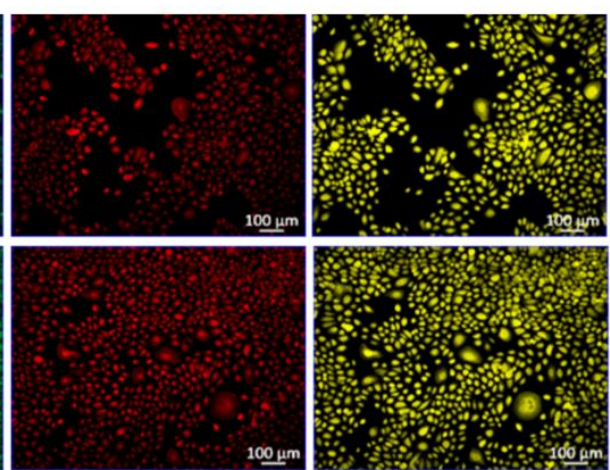

K10

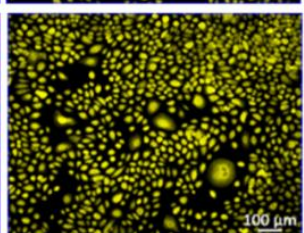

K17

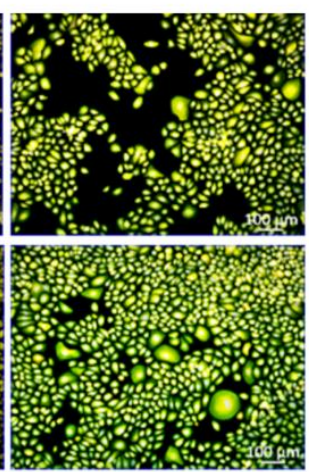

Merge

Supplementary Fig. S3. Effect of 4-AP on cultured keratinocytes. Primary keratinocytes were exposed to 4-AP or no treatment for 24 hours and co-immunostained using antibodies against keratin 14 (proliferation-K14-green), keratin 10 (differentiation-K10-red) or keratin 17 (hyperproliferation-K17-yellow). DAPI (blue) was used as nuclear counterstaining. Scale bars $=$ $100 \mu \mathrm{m}$. 


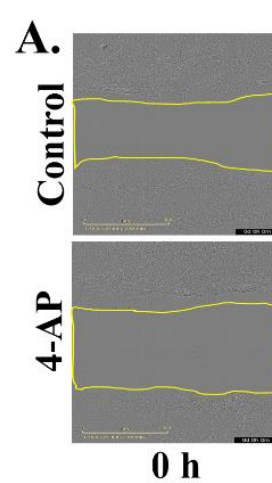

B.

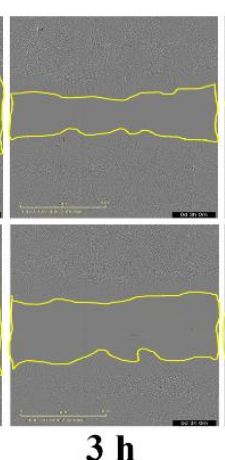

$3 \mathbf{h}$

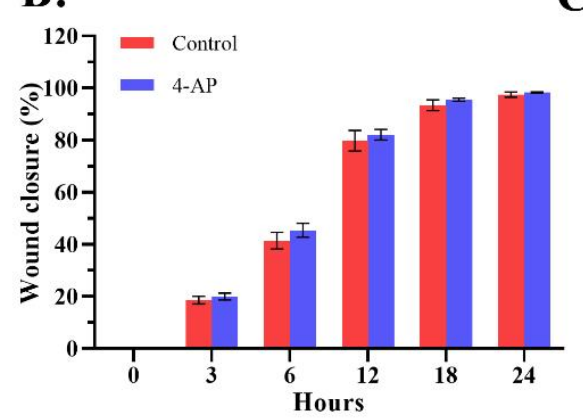

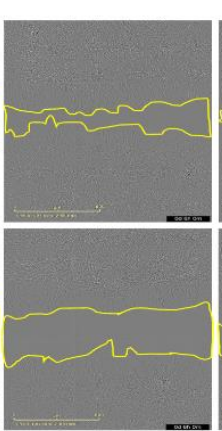

$6 \mathrm{~h}$
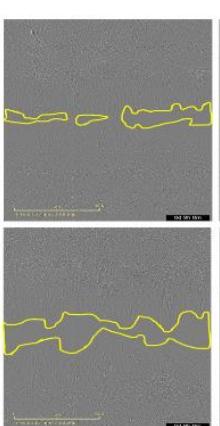

$9 \mathbf{h}$

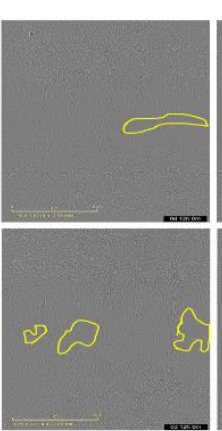

$12 \mathrm{~h}$

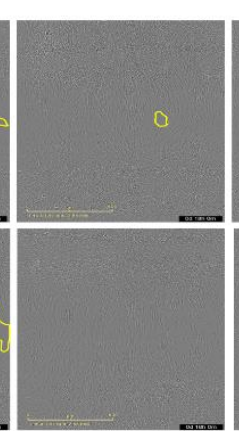

$18 \mathrm{~h}$

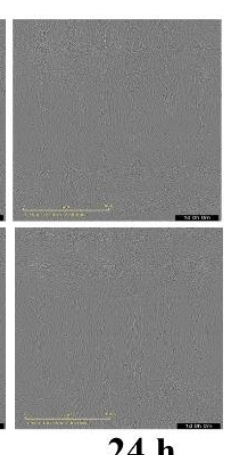

$24 \mathrm{~h}$

C.

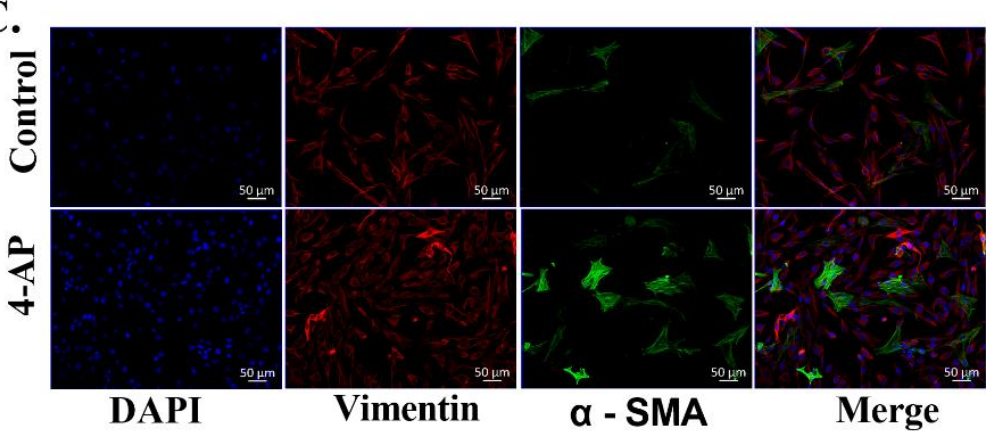

\section{Supplementary Fig. S4. Effect of 4-AP exposure on primary dermal fibroblasts. (A)}

Representative images of in-vitro fibroblast scratch assays with 4-AP and vehicle control at indicated time points. Scale bar $=100 \mu \mathrm{m}$. (B) The relative percentage of wound closure was calculated as the ratio of the remaining wound gap at the given time point compared to time 0 . Data expressed as means $\pm \mathrm{SEM}, \mathrm{N}=4$ wound scratch replicates/group, and $* \mathrm{P}=0.01$ to 0.05 , $* * \mathrm{P}=0.01$ to $0.001, * * * \mathrm{P}<0.0002$, and $* * * * \mathrm{P}<0.0001$ one-way ANOVA Sidak's multiple comparisons test. (C) Co-immunostained dermal fibroblasts exposed to 4-AP or no treatment for 72 hours and immunostained using antibodies against the fibroblast marker vimentin- (red), or the myofibroblast marker $\alpha$-SMA (green). DAPI (blue) was used as nuclear counterstaining. 


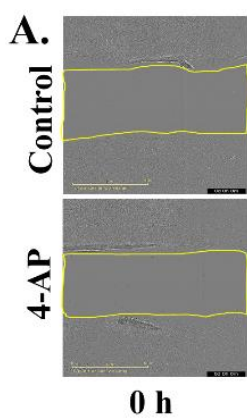

B.

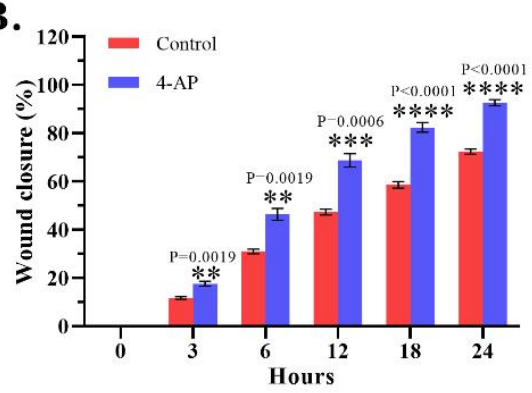

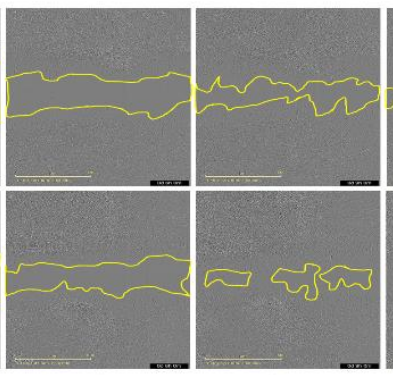

$9 \mathbf{h}$

C. Sox10
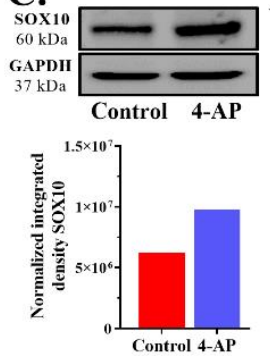

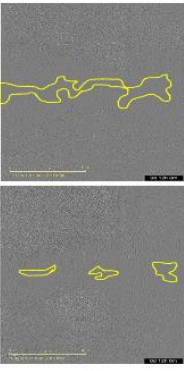

$12 \mathrm{~h}$

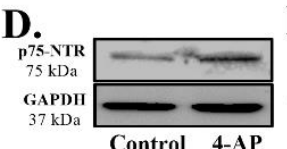

$18 \mathrm{~h}$

E.

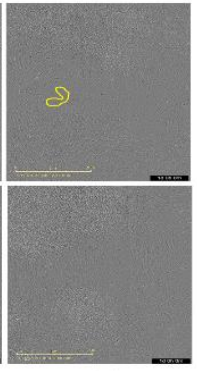

$24 \mathrm{~h}$

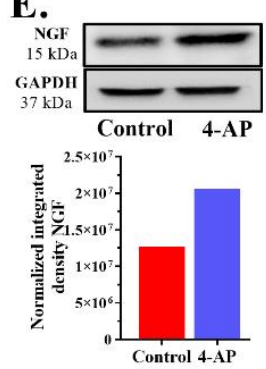

\section{Supplementary Fig. S5. Effect of 4-AP exposure on primary dermal Schwann cell in scratch}

wound healing assays. (A) Representative images of in-vitro Schwann cells scratch assays with 4-AP and vehicle controls at indicated time points. Scale bar $=100 \mu \mathrm{m}$. (B) The relative percentage of wound closure was calculated as the ratio of the remaining wound gap at the given time point compared to time 0 . Mean $\pm \mathrm{SEM}, \mathrm{N}=5$ wound scratch replicates/group, and $* \mathrm{P}=0.01$ to 0.05 , $* * \mathrm{P}=0.01$ to $0.001, * * * \mathrm{P}<0.0002$, and $* * * * \mathrm{P}<0.0001$ one-way ANOVA Sidak's multiple comparisons test. (C) Co-immunostaining of dermal Schwann cells exposed to 4-AP or no treatment for 72 hours and immunostained against SC markers including the Schwann cell marker S100 (green), a de-differentiation marker p75-NTR (red) and myelin basic protein (MBP-yellow). DAPI (blue) was used as nuclear counterstaining. Scale bars, $100 \mu \mathrm{m}$. (D - F) A representative western blot and normalized integrated densities for SOX10, p75-NTR, NGF and GAPDH. 


\section{Supplementary Movies}

Supplementary Movie 1. An example of time-lapse phase contrast images depicting the migration of keratinocytes without treatment (control) during wound scratch closure. Images were recorded every one hour. Scale bar $=100 \mu \mathrm{m}$.

Supplementary Movie 2. An example of time-lapse phase contrast images depicting the migration of keratinocytes after 4-AP treatment during wound scratch closure. Images were recorded every one hour. Scale bar $=100 \mu \mathrm{m}$.

Supplementary Movie 3. An example of time-lapse phase contrast images depicting the migration of fibroblasts without treatment (control) during wound scratch closure. Images were recorded every one hour. Scale bar $=100 \mu \mathrm{m}$.

Supplementary Movie 4. An example of time-lapse phase contrast images depicting the migration of fibroblasts after 4-AP treatment during wound scratch closure. Images were recorded every one hour. Scale bar $=100 \mu \mathrm{m}$.

Supplementary Movie 5. An example of time-lapse phase contrast images depicting the migration of Schwann cells without treatment (control) during wound scratch closure. Images were recorded every one hour. Scale bar $=100 \mu \mathrm{m}$.

Supplementary Movie 6. An example of time-lapse phase contrast images depicting the migration of Schwann cells after 4-AP treatment during wound scratch closure. Images were recorded every one hour. Scale bar $=100 \mu \mathrm{m}$.

Supplementary Movie 7. An example of time-lapse phase contrast images depicting the migration of co-cultured keratinocytes and Schwann cells without treatment (control) during wound scratch closure. Images were recorded every one hour. Scale bar $=100 \mu \mathrm{m}$.

Supplementary Movie 8. An example of time-lapse phase contrast images depicting the migration of co-cultured keratinocytes and Schwann cells after 4-AP treatment during wound scratch closure. Images were recorded every one hour. Scale bar $=100 \mu \mathrm{m}$.

Supplementary Movie 9. An example of time-lapse phase contrast images depicting the migration of co-cultured keratinocytes and fibroblasts without treatment (control) during wound scratch closure. Images were recorded every one hour. Scale bar $=100 \mu \mathrm{m}$. 
Supplementary Movie 10. An example of time-lapse phase contrast images depicting the migration of co-cultured keratinocytes and fibroblasts after 4-AP treatment during wound scratch closure. Images were recorded every one hour. Scale bar $=100 \mu \mathrm{m}$. 